6

\section{SUPPORTING INORMATION}

Impact of Mixture Effects between Emerging Organic Contaminants on Cytotoxicity: A Systems-Biological Understanding of Synergism between TDCPP and TPP

Yingdan Zhang ${ }^{\mathrm{a}, \mathrm{b} \#}$, Min Liu ${ }^{\mathrm{a}, \mathrm{b} \# *}$, Bo Peng ${ }^{\mathrm{a}, \mathrm{b}}$, Shenglan Jia ${ }^{\mathrm{a}, \mathrm{b}}$, Danyu Koh ${ }^{\mathrm{b}}$, Yujue Wang ${ }^{\mathrm{b}}$, Hong Sheng Cheng ${ }^{\mathrm{c}}$, Nguan Soon Tan ${ }^{\mathrm{c}, \mathrm{d}}$, Benedikt Warth ${ }^{\mathrm{e}}$, Da Chen ${ }^{\mathrm{f}}$, Mingliang Fang ${ }^{\mathrm{a}, \mathrm{b}, \mathrm{d}^{*}}$

${ }^{a}$ School of Civil and Environmental Engineering, Nanyang Technological University, Singapore 639798

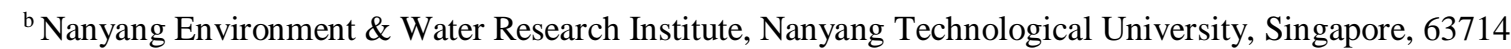

${ }^{\mathrm{c}}$ School of Biological Sciences, Nanyang Technological University Singapore, 60 Nanyang Drive, 637551 Singapore, Singapore

${ }^{\mathrm{d}}$ Lee Kong Chian School of Medicine, Nanyang Technological University Singapore, Clinical Sciences Building, 11 Mandalay Road, 308232 Singapore, Singapore

${ }^{\mathrm{e}}$ University of Vienna, Faculty of Chemistry, Department of Food Chemistry and Toxicology, Währingerstraße 38, 1090 Vienna, Austria

${ }^{\mathrm{f}}$ School of Environment, Jinan University, Guangdong Guangzhou, P. R. China 51144

* Corresponding authors. E-mail address: LIUM0025@e.ntu.edu.sg and mlfang@ntu.edu.sg

\# Authors contributed equally to this manuscript.

This file includes 39 pages, 8 tables, 15 figures and supplemental text. 
37 Table S3. Experimental solubility of compounds in literatures

Table S4. The detailed instrumental parameters

Table S5. Solubility of chemicals in medium and PBS pH $7.4(n=2)$ at $37^{\circ} \mathrm{C}$.

40 Table S6. The detailed information of dysregulated gene and their $\log _{2}$ (Fold Change) value.

41 Table S7. Dysregulated metabolites detected in TDCPP, TPP or Dual samples (Significance criteria: $\mid$ Fold change $(\mathrm{FC}) \mid>=1.5 \& p$-value $=<0.05)$.

Table S8. Joint pathway analysis for integration of metabolomics and transcriptomics data

\section{Figure of Contents}

Figure S1. The determination and calculation steps of combination index (CI) of 78 dual chemical combinations in the chemical interaction screening test (Use $\mathrm{EC}_{50}$ as an example; the mixing ratio for dual chemicals were $\mathrm{m} / \mathrm{n}={ }^{\prime} 1: 4$ ', ' $2: 3$ ', ' $3: 2$ ' or ' $4: 1$ ' in this study; at least 6 times' serial dilution were prepared using growth medium with a diluting factor of 2; the nominal maximal combined dosing concentration is $500 \mu \mathrm{M}$ for each pair)

Figure S2. A to M. Dose-Response curve of 13 selected chemicals fitted using "log[dose]response-inhibition-viable slope: four parameters" model; $\mathrm{x}$-axis represents $\log$ [concentration, $\mu \mathrm{M}]$ and $\mathrm{y}$-axis represents normalized response (\% of maximal response); dash line represents the $95 \%$ confidence interval band; note that carbazole and $\mathrm{B}[\mathrm{a}] \mathrm{p}$ did not reach $50 \%$ inhibition so their $\mathrm{CI}$ values were based on their $\mathrm{EC}_{25}$ values. For single chemical did not exhibit apparent cytotoxicity effect such as BPS and PFOA, their a/A portion was approximated to 0 for calculation.

Figure S3. (1) to (78). Dose-Response curve of 78 dual chemicals combinations with different mixing ratio (' $1: 4$ ', '2:3', ' $3: 2$ ' or ' $4: 1$ ' ) fitted using ' $\log [$ dose]-response-inhibition-viable slope: four parameters" model; $\mathrm{x}$-axis represents $\log [$ concentration, $\mu \mathrm{M}]$ and $\mathrm{y}$-axis represents normalized response (\% of maximal response); curve will be exempted for fitting if a. not follow sigmoidal dose-response relationship; b. no significant response was observed or c. curve bottom is way above/below 0 .

Figure S4. Isobolograms of selected chemical pairs (' $1: 4$ ', ' $2: 3$ ', ' $3: 2$ 'and ' $4: 1$ ') for the inhibition on cell growth of HepG2. A. B[a]p and fluoranthene at $\mathrm{EC}_{25} ; \mathbf{B}$. BADGE and methyl paraben at $\mathrm{EC}_{50} ; \mathbf{C}$. BPA and $\mathrm{BADGE}$ at $\mathrm{EC}_{50} ; \mathbf{D}$. Methyl paraben and carbazole at $\mathrm{EC}_{25}$ and 
interval for the combination ratios indicated. $\equiv \equiv \equiv$ represents additivity line with $95 \%$ confidence belt, the $\mathrm{x}$-axis and $\mathrm{y}$-axis represent the concentrations ( $\mu \mathrm{mol} / \mathrm{L})$ of two chemicals. The area between the two diagonal dotted lines in each plot represents the additive effect; the triangular area under the additive effect region represents the synergistic effect; the area above the additive effect area represent antagonist effect.

Figure S5. Volcano plot illustrating differential expression of genes in Dual versus Control samples.

Figure S6. Volcano plot illustrating differential expression of genes in TDCPP versus Control samples.

Figure S7. Volcano plot illustrating differential expression of genes in TPP versus Control samples.

Figure S8. Classic Venn diagram summarizing the number of shared and distinct GO terms for three treatment conditions.

Figure S9. Gene Ontology (GO) analysis results from DAVID Bioinformatics Resources 6.8 for each treatment conditions A. Dual vs Control; B. TDCPP vs Control; C. TPP vs Control; D. overlapped genes for all treatment conditions

Figure S10. Analysis of dysregulated features in response to TDCPP, TPP and Dual exposure. A. Principal component analysis (PCA) of the dysregulated features for different groups. B. Classic Venn diagram summarizing the number of shared and distinct dysregulated metabolite features for three treatment conditions.

Figure S11. Enrichment analysis of significant metabolic pathways of HepG2 treated with TPP. The data analysis was conducted by the open source platform MetaboAnalyst.

Figure S12. Enrichment analysis of significant metabolic pathways of HepG2 treated with TDCPP. The data analysis was conducted by the open source platform MetaboAnalyst Figure S13. Enrichment analysis of significant metabolic pathways of HepG2 treated with Dual. The data analysis was conducted by the open source platform MetaboAnalyst

4 Figure S14. Dysregulated metabolites mapped to Purine Metabolism and Pyrimidine 5 Metabolism.

96 Figure S15. Dysregulated metabolites and genes expressions in A. "Alanine, aspartate and 7 glutamate metabolism" and B. "Aminoacyl-tRNA biosynthesis" in HepG2 under the 8 exposure of TDCPP, TPP or Dual 
102 Text S1. RNA extraction

103 Text S2. Library preparation, and Illumina Novaseq 6000 Sequencing

104 Text S3. Read mapping

105 Text S4. Differential expression analysis and Functional enrichment

106 Text S5. Solubility and metabolites measurement of compounds in culture medium and PBS

107 buffer. 
109

\begin{tabular}{cccc}
\hline CAS Number & Compound & Experimental Solubility in water $(\mathbf{m g} / \mathbf{1}) 1$ \\
\hline $80-05-7$ & BPA & 120.0 & 112 \\
$1675-54-3$ & BADGE & 1.0 & 113 \\
$80-09-1$ & BPS & 1110.0 & 115 \\
$115-86-6$ & TPP & 1.0 & 116 \\
$13674-87-8$ & TDCPP & 7.0 & 117 \\
$115-96-8$ & TCEP & 7000.0 & 118 \\
207 & Butyl-Paraben & 207.0 & 119 \\
$99-76-3$ & Methyl-Paraben & 2500.0 & 121 \\
$120-47-8$ & Ethyl-Paraben & 885.0 & 122 \\
$86-74-8$ & Carbazole & 1.8 & 123 \\
$50-32-8$ & B[a]p & 0.00162 & 125 \\
$206-44-0$ & Fluoranthene & 0.26 & 126 \\
$335-67-1$ & PFOA & 4340 & 127 \\
& & & 128 \\
& & & 130 \\
\hline
\end{tabular}

132 Note: TPP, TDCPP, B[a]p, fluoranthene, carbazole, PFOA and BADGE may have saturated 133 concentrations according to the experimental solubility. Therefore, these chemicals were conducted 134 further solubility test in culture medium. 
137 Table S4. The detailed instrumental parameters

138

\begin{tabular}{|c|c|c|c|c|c|}
\hline Analytes & Instrument & Parent Ion & $\begin{array}{l}\text { Retention } \\
\text { Time }\end{array}$ & Mode & Internal Standard \\
\hline $\mathrm{BDCPP}$ & \multirow{7}{*}{$\begin{array}{c}\text { LC-QToF- } \\
\text { MS }\end{array}$} & 318.9221 & 5.238 & \multirow[t]{5}{*}{$\mathrm{ESI}(+)$} & DPP-d10 \\
\hline TDCPP & & 428.8911 & 8.139 & & BDCIPP-d12 \\
\hline $\mathrm{TPP}$ & & 327.078 & 8.371 & & TCEP-d12 \\
\hline DPP & & 251.04 & 4.97 & & DPP-d10 \\
\hline BADGE & & 358.2016 & 8.185 & & BADGE-d6 \\
\hline PFOA & & 412.9664 & 6.3 & ESI(-) & PFOA- ${ }^{13} \mathrm{C} 4$ \\
\hline TPP-OH & & 344.0808 & 7 & $\mathrm{ESI}(+)$ & DPP-d10 \\
\hline $\mathrm{B}[\mathrm{a}] \mathrm{p}$ & \multirow[t]{3}{*}{ GC-MS } & 253 & 15.04 & & Chrysene-d12 \\
\hline Fluoranthene & & 202.8 & 10.36 & & \multirow[b]{2}{*}{ Phenanthrene-d10 } \\
\hline Carbazole & & 167.6 & 8.8 & & \\
\hline
\end{tabular}

139 Note: Instrumental parameters for LC-QToF-MS: Capillary voltage $3500 \mathrm{~V}$; fragmentor $175 \mathrm{~V}$; gas

140 temperature $225^{\circ} \mathrm{C}$; dry gas $14 \mathrm{~L} / \mathrm{min}$ and nebulizer 35 psi. Full-scan data acquisition was performed

141 by scanning from $\mathrm{m} / \mathrm{z} 50$ to 1000 . 
144 Table S5. Solubility of chemicals in medium and PBS pH $7.4(\mathrm{n}=2)$ at $37^{\circ} \mathrm{C}$.

\begin{tabular}{|c|c|c|c|c|c|}
\hline \multirow[t]{2}{*}{ Chemical } & \multirow[t]{2}{*}{ Solvent } & \multicolumn{2}{|c|}{ Actual Concentration } & \multicolumn{2}{|c|}{ Nominal Concentration } \\
\hline & & $(\mu \mathrm{g} / \mathrm{mL})$ & $(\mu \mathrm{M})$ & $(\mu \mathrm{g} / \mathrm{mL})$ & $(\mu \mathrm{M})$ \\
\hline \multirow[t]{2}{*}{ Total BADGE $^{\mathrm{a}}$} & Medium & $34.0 \pm 0.3$ & $100.0 \pm 0.9$ & 34.0 & 100 \\
\hline & PBS & $34.0 \pm 0.4$ & $100.0 \pm 1.0$ & & \\
\hline \multirow[t]{2}{*}{ TPP } & Medium & $22.7 \pm 2.1$ & $69.6 \pm 6.4$ & 32.6 & 100 \\
\hline & PBS & $4.2 \pm 0.6$ & $12.9 \pm 1.8$ & & \\
\hline \multirow[t]{2}{*}{ TDCPP } & Medium & $38.6 \pm 0.3$ & $89.6 \pm 0.7$ & 43.0 & 100 \\
\hline & PBS & $39.1 \pm 3.6$ & $90.7 \pm 8.4$ & & \\
\hline \multirow[t]{2}{*}{$\mathrm{B}[\mathrm{a}] \mathrm{P}$} & Medium & $6.1 \pm 0.4$ & $24.0 \pm 1.6$ & 6.3 & 25 \\
\hline & PBS & $<\mathrm{LOQ}$ & & & \\
\hline \multirow[t]{2}{*}{ Fluoranthene } & Medium & $11.8 \pm 0.1$ & $58.3 \pm 0.5$ & 20.2 & 100 \\
\hline & PBS & $0.8 \pm 0.6$ & $3.9 \pm 2.9$ & & \\
\hline \multirow[t]{2}{*}{ Carbazole } & Medium & $13.1 \pm 0.1$ & $78.3 \pm 0.6$ & 16.7 & 100 \\
\hline & PBS & $8.3 \pm 0.7$ & $49.6 \pm 4.2$ & & \\
\hline
\end{tabular}

145 Notes:

$146{ }^{a}$ The value reported here is total BADGEs including BADGE itself and its two hydrolysis

147 products $\mathrm{BADGE}-\mathrm{H}_{2} \mathrm{O}$ and $\mathrm{BADGE}-2 \mathrm{H}_{2} \mathrm{O}$; BADGE will be readily hydrolysed to BADGE-

$148 \mathrm{H}_{2} \mathrm{O}$ and BADGE-2 $\mathrm{H}_{2} \mathrm{O}$ when water presents. The detected actual concentration of BADGE is

$1495.1 \pm 0.3 \mu \mathrm{g} / \mathrm{mL}$ and $5.0 \pm 0.5 \mu \mathrm{g} / \mathrm{mL}$ in medium and PBS respectively. 
150 Table S6. The detailed information of dysregulated gene and their $\log _{2}$ (Fold Change) value.

\begin{tabular}{|c|c|c|c|c|}
\hline \multirow{2}{*}{ ENSEMBL_GENE_ID } & \multirow{2}{*}{ Gene_name } & \multicolumn{3}{|c|}{$\log _{2}($ Fold Change $)$} \\
\hline & & Dual & TDCPP & TPP \\
\hline ENSG00000001630 & CYP51A1 & 0.54 & 0.22 & 0.41 \\
\hline ENSG00000004799 & PDK4 & 1.39 & 0.38 & 1.13 \\
\hline ENSG00000005884 & ITGA3 & 0.55 & 0.10 & 0.24 \\
\hline ENSG00000006007 & GDE1 & 0.45 & 0.09 & 0.15 \\
\hline ENSG00000040275 & SPDL1 & -0.29 & -0.23 & -0.16 \\
\hline ENSG00000042445 & RETSAT & 0.58 & 0.20 & 0.32 \\
\hline ENSG00000044574 & HSPA5 & 0.59 & 0.37 & 0.16 \\
\hline ENSG00000051108 & HERPUD1 & 1.23 & 0.78 & 0.34 \\
\hline ENSG00000052802 & MSMO1 & 1.29 & 0.73 & 1.19 \\
\hline ENSG00000062282 & DGAT2 & -0.57 & -0.55 & -0.30 \\
\hline ENSG00000066135 & KDM4A & 0.35 & 0.15 & 0.05 \\
\hline ENSG00000067064 & IDI1 & 0.75 & 0.46 & 0.73 \\
\hline ENSG00000067182 & TNFRSF1A & 0.41 & 0.22 & 0.19 \\
\hline ENSG00000067334 & DNTTIP2 & -0.49 & -0.28 & -0.03 \\
\hline ENSG00000070669 & ASNS & 0.56 & 0.30 & 0.13 \\
\hline ENSG00000072310 & SREBF1 & 0.44 & 0.04 & 0.42 \\
\hline ENSG00000072415 & MPP5 & -0.30 & -0.21 & -0.14 \\
\hline ENSG00000077238 & IL4R & 0.51 & 0.86 & 0.27 \\
\hline ENSG00000078401 & EDN1 & -0.73 & -0.60 & -0.26 \\
\hline ENSG00000079459 & FDFT1 & 0.60 & 0.32 & 0.46 \\
\hline ENSG00000084710 & EFR3B & 0.92 & 0.53 & 0.39 \\
\hline ENSG00000086758 & HUWE1 & 0.59 & 0.20 & 0.15 \\
\hline ENSG00000090861 & AARS & 0.55 & 0.21 & 0.12 \\
\hline ENSG00000092140 & G2E3 & -0.39 & -0.28 & -0.09 \\
\hline ENSG00000092621 & PHGDH & 0.44 & 0.16 & 0.03 \\
\hline ENSG00000092964 & DPYSL2 & 0.67 & 0.23 & 0.16 \\
\hline ENSG00000099194 & SCD & 1.17 & 0.46 & 0.73 \\
\hline ENSG00000100219 & XBP1 & 0.78 & 0.53 & 0.28 \\
\hline ENSG00000100330 & MTMR3 & 0.48 & 0.24 & 0.09 \\
\hline ENSG00000100889 & PCK2 & 0.59 & 0.22 & -0.04 \\
\hline ENSG00000101236 & RNF24 & 0.49 & 0.25 & 0.13 \\
\hline ENSG00000101255 & TRIB3 & 0.84 & 0.43 & 0.18 \\
\hline ENSG00000101577 & LPIN2 & 0.75 & 0.29 & 0.15 \\
\hline ENSG00000102580 & DNAJC3 & 0.61 & 0.25 & 0.17 \\
\hline ENSG00000103657 & HERC1 & 0.70 & 0.11 & 0.35 \\
\hline ENSG00000104549 & SQLE & 0.88 & 0.41 & 0.62 \\
\hline ENSG00000105821 & DNAJC2 & -0.32 & -0.20 & -0.12 \\
\hline ENSG00000105856 & HBP1 & 0.43 & 0.43 & 0.07 \\
\hline ENSG00000106105 & GARS & 0.48 & 0.29 & 0.12 \\
\hline
\end{tabular}




\begin{tabular}{|c|c|c|c|c|}
\hline ENSG00000106665 & CLIP2 & 0.68 & 0.19 & 0.11 \\
\hline ENSG00000107263 & RAPGEF1 & 0.48 & 0.28 & 0.07 \\
\hline ENSG00000107554 & DNMBP & 0.52 & 0.14 & 0.02 \\
\hline ENSG00000107863 & ARHGAP21 & 0.60 & 0.32 & 0.26 \\
\hline ENSG00000108846 & $\mathrm{ABCC} 3$ & 0.59 & 0.12 & 0.17 \\
\hline ENSG00000108932 & SLC16A6 & 0.43 & 0.41 & 0.22 \\
\hline ENSG00000110048 & OSBP & 0.48 & 0.24 & 0.09 \\
\hline ENSG00000110619 & CARS & 0.61 & 0.36 & 0.15 \\
\hline ENSG00000110921 & MVK & 0.89 & 0.42 & 0.84 \\
\hline ENSG00000112081 & SRSF3 & -0.44 & -0.36 & -0.10 \\
\hline ENSG00000113161 & HMGCR & 0.70 & 0.20 & 0.51 \\
\hline ENSG00000113739 & STC2 & 0.66 & 0.45 & 0.16 \\
\hline ENSG00000114796 & KLHL24 & -0.14 & -0.61 & -0.73 \\
\hline ENSG00000115183 & TANC1 & 0.50 & 0.23 & 0.20 \\
\hline ENSG00000115368 & WDR75 & -0.38 & -0.22 & -0.16 \\
\hline ENSG00000115875 & SRSF7 & -0.58 & -0.38 & -0.15 \\
\hline ENSG00000115902 & SLC1A4 & 0.67 & 0.40 & 0.19 \\
\hline ENSG00000116016 & EPAS1 & 0.41 & 0.11 & 0.40 \\
\hline ENSG00000116133 & DHCR24 & 0.52 & 0.15 & 0.25 \\
\hline ENSG00000116285 & ERRFI1 & 0.60 & 0.33 & 0.16 \\
\hline ENSG00000116761 & $\mathrm{CTH}$ & 0.63 & 0.51 & 0.22 \\
\hline ENSG00000118418 & HMGN3 & -0.40 & -0.17 & -0.14 \\
\hline ENSG00000120129 & DUSP1 & 0.43 & 0.30 & 0.14 \\
\hline ENSG00000120137 & PANK3 & 0.41 & 0.20 & 0.38 \\
\hline ENSG00000120738 & EGR1 & 1.16 & 0.75 & 0.33 \\
\hline ENSG00000120837 & NFYB & -0.29 & -0.14 & 0.01 \\
\hline ENSG00000120875 & DUSP4 & 0.68 & 0.38 & 0.34 \\
\hline ENSG00000122218 & COPA & 0.42 & 0.17 & 0.05 \\
\hline ENSG00000122778 & KIAA1549 & 0.53 & 0.15 & 0.14 \\
\hline ENSG00000123983 & ACSL3 & 0.36 & 0.22 & 0.32 \\
\hline ENSG00000124762 & CDKN1A & 0.72 & 0.48 & 0.30 \\
\hline ENSG00000125378 & BMP4 & -0.80 & -0.59 & -0.49 \\
\hline ENSG00000125657 & TNFSF9 & 0.18 & 0.38 & 0.14 \\
\hline ENSG00000127481 & UBR4 & 0.63 & 0.26 & 0.22 \\
\hline ENSG00000127989 & MTERF1 & -0.60 & -0.31 & -0.17 \\
\hline ENSG00000128165 & $\mathrm{ADM} 2$ & 2.20 & 1.74 & 1.15 \\
\hline ENSG00000128272 & ATF4 & 0.86 & 0.71 & 0.27 \\
\hline ENSG00000128590 & DNAJB9 & 1.12 & 0.92 & 0.65 \\
\hline ENSG00000128965 & CHAC1 & 2.44 & 1.70 & 0.82 \\
\hline ENSG00000129566 & TEP1 & 0.33 & -0.11 & 0.02 \\
\hline ENSG00000130164 & LDLR & 1.51 & 0.72 & 1.02 \\
\hline ENSG00000130513 & GDF15 & 0.87 & 0.68 & 0.25 \\
\hline
\end{tabular}




\begin{tabular}{|c|c|c|c|c|}
\hline ENSG00000130522 & JUND & 0.47 & 0.32 & 0.28 \\
\hline ENSG00000130766 & SESN2 & 1.47 & 0.87 & 0.38 \\
\hline ENSG00000131018 & SYNE1 & 0.61 & 0.32 & 0.17 \\
\hline ENSG00000131069 & ACSS2 & 0.58 & 0.28 & 0.08 \\
\hline ENSG00000131389 & SLC6A6 & 0.59 & 0.38 & 0.27 \\
\hline ENSG00000132196 & HSD17B7 & 0.67 & 0.36 & 0.32 \\
\hline ENSG00000133639 & BTG1 & 0.37 & 0.36 & 0.26 \\
\hline ENSG00000133641 & C12orf29 & -0.29 & -0.02 & -0.06 \\
\hline ENSG00000134107 & BHLHE40 & 0.72 & 0.39 & 0.41 \\
\hline ENSG00000134186 & PRPF38B & -0.25 & -0.18 & -0.09 \\
\hline ENSG00000134294 & SLC38A2 & 0.67 & 0.51 & 0.26 \\
\hline ENSG00000134324 & LPIN1 & 0.89 & 0.31 & 0.55 \\
\hline ENSG00000134684 & YARS & 0.47 & 0.25 & 0.16 \\
\hline ENSG00000135842 & FAM129A & 0.49 & 0.06 & 0.11 \\
\hline ENSG00000136826 & KLF4 & 0.48 & 0.32 & 0.41 \\
\hline ENSG00000137193 & PIM1 & 0.75 & 0.53 & 0.18 \\
\hline ENSG00000137331 & IER3 & 0.53 & 0.48 & 0.21 \\
\hline ENSG00000138398 & PPIG & -0.41 & -0.28 & -0.08 \\
\hline ENSG00000138764 & $\mathrm{CCNG} 2$ & 0.57 & 0.31 & -0.04 \\
\hline ENSG00000139269 & INHBE & 1.28 & 0.60 & 0.18 \\
\hline ENSG00000139289 & PHLDA1 & 1.01 & 0.66 & 0.63 \\
\hline ENSG00000139318 & DUSP6 & 1.68 & 0.51 & 0.51 \\
\hline ENSG00000139725 & RHOF & 0.38 & 0.08 & 0.15 \\
\hline ENSG00000140044 & JDP2 & 0.50 & 0.33 & 0.05 \\
\hline ENSG00000141458 & NPC1 & 0.47 & 0.12 & 0.11 \\
\hline ENSG00000144136 & SLC20A1 & 0.63 & 0.42 & 0.28 \\
\hline ENSG00000144824 & PHLDB2 & 0.47 & 0.27 & 0.29 \\
\hline ENSG00000146072 & TNFRSF21 & 0.77 & 0.33 & 0.49 \\
\hline ENSG00000146674 & IGFBP3 & -0.94 & -0.70 & -0.43 \\
\hline ENSG00000148841 & ITPRIP & 0.72 & 0.47 & 0.32 \\
\hline ENSG00000148926 & $\mathrm{ADM}$ & -0.43 & -0.32 & -0.16 \\
\hline ENSG00000149428 & HYOU1 & 0.48 & 0.27 & 0.09 \\
\hline ENSG00000150961 & SEC24D & 0.66 & 0.41 & 0.16 \\
\hline ENSG00000150995 & ITPR1 & 0.54 & 0.35 & 0.22 \\
\hline ENSG00000151012 & SLC7A11 & 0.40 & 0.12 & 0.16 \\
\hline ENSG00000154127 & UBASH3B & 0.76 & -0.06 & 0.27 \\
\hline ENSG00000155100 & OTUD6B & -0.54 & -0.27 & -0.23 \\
\hline ENSG00000156650 & KAT6B & 0.56 & 0.38 & 0.18 \\
\hline ENSG00000158246 & FAM46B & -0.49 & -0.32 & -0.28 \\
\hline ENSG00000159921 & GNE & 0.55 & 0.27 & 0.30 \\
\hline ENSG00000160285 & LSS & 0.71 & 0.34 & 0.49 \\
\hline ENSG00000161011 & SQSTM1 & 0.40 & 0.18 & 0.27 \\
\hline
\end{tabular}




\begin{tabular}{|c|c|c|c|c|}
\hline ENSG00000163347 & CLDN1 & 0.47 & 0.22 & 0.24 \\
\hline ENSG00000163961 & RNF168 & 0.67 & 0.21 & 0.14 \\
\hline ENSG00000164171 & ITGA2 & 0.81 & 0.35 & 0.45 \\
\hline ENSG00000164211 & STARD4 & 1.16 & 0.67 & 0.76 \\
\hline ENSG00000165030 & NFIL3 & 0.73 & 0.49 & 0.34 \\
\hline ENSG00000165494 & PCF11 & -0.42 & -0.23 & -0.18 \\
\hline ENSG00000165525 & NEMF & -0.43 & -0.18 & -0.09 \\
\hline ENSG00000166750 & SLFN5 & 0.57 & 0.27 & 0.21 \\
\hline ENSG00000166783 & MARF1 & 0.69 & 0.47 & 0.24 \\
\hline ENSG00000167508 & MVD & 0.86 & 0.61 & 0.74 \\
\hline ENSG00000167676 & PLIN4 & 2.54 & 0.93 & 1.02 \\
\hline ENSG00000167772 & ANGPTL4 & 1.76 & 0.19 & 1.98 \\
\hline ENSG00000168003 & SLC3A2 & 0.37 & 0.12 & 0.09 \\
\hline ENSG00000168016 & TRANK1 & 0.84 & 0.56 & 0.29 \\
\hline ENSG00000168209 & DDIT4 & 1.05 & 0.60 & 0.23 \\
\hline ENSG00000168264 & IRF2BP2 & 0.34 & 0.35 & 0.24 \\
\hline ENSG00000168453 & $\mathrm{HR}$ & 0.53 & 0.12 & 0.42 \\
\hline ENSG00000168685 & IL7R & -0.36 & -0.42 & 0.01 \\
\hline ENSG00000169020 & ATP5I & -0.44 & -0.11 & -0.08 \\
\hline ENSG00000169174 & PCSK9 & 0.65 & 0.30 & 0.32 \\
\hline ENSG00000169710 & FASN & 0.58 & -0.01 & 0.24 \\
\hline ENSG00000170385 & SLC30A1 & 0.42 & 0.22 & 0.28 \\
\hline ENSG00000170776 & AKAP13 & 0.39 & -0.02 & 0.11 \\
\hline ENSG00000171303 & $\mathrm{KCNK} 3$ & 0.54 & 0.11 & 0.28 \\
\hline ENSG00000171940 & $\mathrm{ZNF} 217$ & 0.50 & 0.17 & 0.08 \\
\hline ENSG00000172071 & EIF2AK3 & 0.83 & 0.65 & 0.35 \\
\hline ENSG00000172216 & CEBPB & 0.27 & 0.44 & 0.36 \\
\hline ENSG00000172493 & AFF1 & 0.54 & 0.16 & 0.08 \\
\hline ENSG00000172780 & RAB43 & 0.22 & 0.01 & 0.24 \\
\hline ENSG00000172893 & DHCR7 & 0.58 & 0.15 & 0.36 \\
\hline ENSG00000173166 & RAPH1 & 0.36 & 0.11 & 0.11 \\
\hline ENSG00000173391 & OLR1 & -0.38 & -0.41 & -0.13 \\
\hline ENSG00000173575 & CHD2 & 0.58 & 0.46 & 0.37 \\
\hline ENSG00000174173 & TRMT10C & -0.47 & -0.24 & -0.06 \\
\hline ENSG00000175197 & DDIT3 & 0.87 & 0.65 & 0.23 \\
\hline ENSG00000175832 & ETV4 & 0.51 & 0.17 & 0.22 \\
\hline ENSG00000176046 & NUPR1 & 0.88 & 0.71 & 0.38 \\
\hline ENSG00000176124 & DLEU1 & -0.44 & -0.28 & -0.14 \\
\hline ENSG00000176700 & SCAND2P & 1.16 & 1.19 & 1.13 \\
\hline ENSG00000177169 & ULK1 & 0.56 & 0.33 & 0.05 \\
\hline ENSG00000177409 & SAMD9L & -0.56 & -0.43 & -0.39 \\
\hline ENSG00000179562 & $\mathrm{GCC} 1$ & 0.57 & 0.09 & 0.10 \\
\hline
\end{tabular}




\begin{tabular}{|c|c|c|c|c|}
\hline ENSG00000181789 & COPG1 & 0.46 & 0.13 & 0.09 \\
\hline ENSG00000182010 & RTKN2 & -0.53 & -0.36 & -0.32 \\
\hline ENSG00000182158 & CREB3L2 & 0.64 & 0.34 & 0.27 \\
\hline ENSG00000183801 & OLFML1 & -0.42 & -0.39 & -0.40 \\
\hline ENSG00000184677 & ZBTB40 & 0.53 & 0.22 & 0.21 \\
\hline ENSG00000185022 & MAFF & 0.54 & 0.48 & 0.31 \\
\hline ENSG00000185262 & UBALD2 & 0.35 & 0.42 & 0.07 \\
\hline ENSG00000186480 & INSIG1 & 1.59 & 0.73 & 1.20 \\
\hline ENSG00000186918 & ZNF395 & 0.59 & 0.28 & 0.13 \\
\hline ENSG00000188064 & WNT7B & -0.38 & -0.33 & -0.28 \\
\hline ENSG00000188290 & HES4 & -0.49 & -0.12 & -0.03 \\
\hline ENSG00000189057 & FAM111B & -0.52 & -0.33 & -0.10 \\
\hline ENSG00000196182 & STK40 & 0.62 & 0.26 & 0.16 \\
\hline ENSG00000196950 & SLC39A10 & -0.34 & -0.15 & -0.12 \\
\hline ENSG00000198380 & GFPT1 & 0.45 & 0.22 & 0.10 \\
\hline ENSG00000198911 & SREBF2 & 0.51 & 0.12 & 0.17 \\
\hline ENSG00000198952 & SMG5 & 0.33 & 0.11 & 0.14 \\
\hline ENSG00000203668 & CHML & -0.39 & -0.34 & -0.19 \\
\hline ENSG00000204388 & HSPA1B & 0.69 & 0.25 & 0.43 \\
\hline ENSG00000204389 & HSPA1A & 0.54 & 0.32 & 0.46 \\
\hline ENSG00000205336 & ADGRG1 & 0.72 & 0.25 & 0.46 \\
\hline ENSG00000205609 & EIF3CL & 0.45 & 0.32 & 0.51 \\
\hline ENSG00000213741 & RPS29 & -0.35 & -0.08 & -0.07 \\
\hline ENSG00000214510 & SPINK13 & -0.86 & -0.09 & -0.07 \\
\hline ENSG00000225855 & RUSC1-AS1 & 0.91 & 0.28 & 0.52 \\
\hline ENSG00000228106 & AL392172.1 & -0.79 & -0.50 & -0.14 \\
\hline ENSG00000228830 & AL160408.2 & 0.93 & 0.26 & 0.13 \\
\hline ENSG00000231412 & AC005392.2 & 0.59 & 0.31 & 0.25 \\
\hline ENSG00000236144 & $\begin{array}{l}\text { TMEM147- } \\
\text { AS1 }\end{array}$ & -0.58 & -0.48 & -0.22 \\
\hline ENSG00000240053 & LY6G5B & -0.30 & -0.95 & -0.18 \\
\hline ENSG00000240344 & PPIL3 & -0.39 & -0.14 & -0.16 \\
\hline ENSG00000244165 & P2RY11 & -0.87 & -0.79 & -0.61 \\
\hline ENSG00000244405 & ETV5 & 0.38 & 0.43 & 0.12 \\
\hline ENSG00000247844 & CCAT1 & -0.63 & -0.84 & -0.47 \\
\hline ENSG00000251201 & $\begin{array}{l}\text { TMED7- } \\
\text { TICAM2 }\end{array}$ & 0.61 & 0.77 & 0.37 \\
\hline ENSG00000254721 & AP000879.1 & -1.11 & -0.50 & 0.16 \\
\hline ENSG00000257315 & ZBED6 & 0.33 & 0.39 & 0.28 \\
\hline ENSG00000258539 & AC068896.1 & 1.16 & 0.54 & 0.40 \\
\hline ENSG00000259024 & $\begin{array}{c}\text { TVP23C- } \\
\text { CDRT4 }\end{array}$ & 0.81 & 0.55 & 0.24 \\
\hline ENSG00000259332 & ST20-MTHFS & -0.61 & -0.58 & -0.18 \\
\hline ENSG00000263155 & MYZAP & 1.03 & 0.67 & 0.79 \\
\hline
\end{tabular}




\begin{tabular}{|c|c|c|c|c|} 
ENSG00000264772 & AC016876.2 & -0.23 & -0.58 & -0.25 \\
\hline ENSG00000266094 & RASSF5 & 0.62 & 0.35 & 0.23 \\
\hline ENSG00000275496 & CU633906.1 & 1.14 & -0.08 & 1.08 \\
\hline ENSG00000275740 & AC091959.3 & 1.19 & -0.08 & 0.26 \\
\hline ENSG00000276850 & AC245041.3 & -0.19 & -0.23 & -0.11 \\
\hline ENSG00000278599 & TBC1D3E & -1.27 & -0.59 & -1.08 \\
\hline ENSG00000281383 & FP671120.4 & 0.84 & 0.80 & 0.47 \\
\hline ENSG00000282914 & Z83818.2 & -0.73 & -0.34 & -0.50 \\
\hline ENSG00000283765 & AC131160.1 & 0.53 & 0.91 & 0.95 \\
\hline
\end{tabular}

151

152

153 
154 Table S7. Dysregulated metabolites detected in TDCPP, TPP or Dual samples (Significance criteria:

$155 \mid$ Fold change(FC) $\mid>=1.5 \& p$-value $=<0.05)$.

\begin{tabular}{|c|c|c|c|c|c|c|c|c|c|c|}
\hline \multirow[t]{2}{*}{ Name } & \multirow{2}{*}{$\begin{array}{l}\text { KEGG or } \\
\text { HMDB }\end{array}$} & \multicolumn{3}{|c|}{ TDCPP } & \multicolumn{3}{|c|}{ TPP } & \multicolumn{3}{|l|}{ Dual } \\
\hline & & FC & SD & $p$ & FC & SD & $p$ & FC & SD & $p$ \\
\hline ATP & $\mathrm{C} 00002$ & 0.5 & 0.1 & 0.0 & 0.9 & 0.1 & 0.4 & 0.4 & 0.1 & 0.0 \\
\hline NADH & $\mathrm{C} 00004$ & 1.5 & 0.2 & 0.0 & 1.4 & 0.2 & 0.0 & 0.6 & 0.1 & 0.0 \\
\hline $\mathrm{ADP}$ & $\mathrm{C} 00008$ & 0.6 & 0.1 & 0.0 & 0.6 & 0.1 & 0.0 & 0.5 & 0.1 & 0.0 \\
\hline UDP & $\mathrm{C} 00015$ & 0.5 & 0.1 & 0.0 & 0.6 & 0.1 & 0.0 & 0.3 & 0.1 & 0.0 \\
\hline AMP & $\mathrm{C} 00020$ & 0.5 & 0.0 & 0.0 & 0.9 & 0.0 & 0.0 & 0.3 & 0.2 & 0.0 \\
\hline Glutamate & $\mathrm{C} 00025$ & 0.7 & 0.0 & 0.0 & 0.9 & 0.1 & 0.0 & 0.5 & 0.0 & 0.0 \\
\hline UDP-glucose & $\mathrm{C} 00029$ & 0.5 & 0.1 & 0.0 & 0.8 & 0.0 & 0.3 & 0.3 & 0.0 & 0.0 \\
\hline GDP & $\mathrm{C} 00035$ & 0.5 & 0.1 & 0.0 & 0.6 & 0.1 & 0.0 & 0.3 & 0.0 & 0.0 \\
\hline Glycine & C00037 & 0.7 & 0.1 & 0.0 & 0.8 & 0.1 & 0.0 & 0.4 & 0.1 & 0.0 \\
\hline Succinate & $\mathrm{C} 00042$ & 0.7 & 0.2 & 0.0 & 0.7 & 0.0 & 0.0 & 1.0 & 0.5 & 1.0 \\
\hline $\begin{array}{c}\text { UDP-N- } \\
\text { acetyglucosamine }\end{array}$ & $\mathrm{C} 00043$ & 0.7 & 0.2 & 0.0 & 0.8 & 0.1 & 0.1 & 0.5 & 0.2 & 0.0 \\
\hline Lysine & $\mathrm{C} 00047$ & 1.2 & 0.2 & 0.2 & 0.9 & 0.1 & 0.6 & 0.8 & 0.1 & 0.4 \\
\hline Asparate & $\mathrm{C} 00049$ & 0.4 & 0.1 & 0.0 & 0.6 & 0.1 & 0.0 & 0.3 & 0.1 & 0.0 \\
\hline Glutathione & $\mathrm{C} 00051$ & 0.5 & 0.1 & 0.0 & 0.9 & 0.1 & 0.3 & 0.2 & 0.2 & 0.0 \\
\hline CMP & $\mathrm{C} 00055$ & 0.4 & 0.0 & 0.0 & 0.8 & 0.1 & 0.0 & 0.2 & 0.1 & 0.0 \\
\hline CTP & $\mathrm{C} 00063$ & 0.5 & 0.1 & 0.0 & 0.9 & 0.0 & 0.4 & 0.3 & 0.0 & 0.0 \\
\hline Glutamine & $\mathrm{C} 00064$ & 0.8 & 0.1 & 0.1 & 0.8 & 0.1 & 0.0 & 0.6 & 0.1 & 0.0 \\
\hline Serine & $\mathrm{C} 00065$ & 0.9 & 0.1 & 0.3 & 1.0 & 0.2 & 1.0 & 0.7 & 0.1 & 0.0 \\
\hline UTP & $\mathrm{C} 00075$ & 0.5 & 0.1 & 0.0 & 0.9 & 0.1 & 0.4 & 0.3 & 0.0 & 0.0 \\
\hline Ornithine & $\mathrm{C} 00077$ & 1.2 & 0.2 & 0.4 & 0.9 & 0.1 & 0.5 & 1.0 & 0.2 & 0.9 \\
\hline Tryptophan & $\mathrm{C} 00078$ & 1.1 & 0.1 & 0.7 & 1.0 & 0.0 & 0.9 & 0.7 & 0.2 & 0.1 \\
\hline Phenylalanine & C00079 & 0.8 & 0.5 & 0.7 & 0.8 & 0.4 & 0.7 & 0.4 & 0.1 & 0.3 \\
\hline Uracil & C00106 & 2.0 & 0.7 & 0.1 & 0.8 & 0.4 & 0.7 & 49.8 & 62.7 & 0.2 \\
\hline $\begin{array}{l}\text { Dihydroxy-acetone- } \\
\text { phosphate }\end{array}$ & $\mathrm{C} 00111$ & 1.0 & 0.1 & 0.8 & 1.3 & 0.2 & 0.1 & 3.3 & 3.5 & 0.3 \\
\hline Fumarate & $\mathrm{C} 00122$ & 0.7 & 0.2 & 0.0 & 1.0 & 0.1 & 0.7 & 0.4 & 0.3 & 0.0 \\
\hline Leucine & C00123 & 1.0 & 0.2 & 0.8 & 1.0 & 0.1 & 1.0 & 0.8 & 0.1 & 0.3 \\
\hline Glutathione disulfide & $\mathrm{C} 00127$ & 0.5 & 0.0 & 0.1 & 0.9 & 0.2 & 0.4 & 0.6 & 0.3 & 0.1 \\
\hline Histidine & $\mathrm{C} 00135$ & 0.9 & 0.3 & 0.4 & 1.1 & 0.1 & 0.2 & 0.9 & 0.1 & 0.2 \\
\hline Adenine & $\mathrm{C} 00147$ & 1.0 & 0.2 & 0.9 & 1.0 & 0.3 & 0.8 & 0.5 & 0.1 & 0.0 \\
\hline Asparagine & $\mathrm{C} 00152$ & 1.1 & 0.2 & 0.5 & 1.0 & 0.1 & 1.0 & 1.1 & 0.4 & 0.6 \\
\hline Citric acid & $\mathrm{C} 00158$ & 0.3 & 0.0 & 0.0 & 0.6 & 0.1 & 0.0 & 0.7 & 0.6 & 0.3 \\
\hline UDP-Glucuronate & $\mathrm{C} 00167$ & 0.7 & 0.1 & 0.0 & 1.0 & 0.1 & 0.9 & 0.5 & 0.1 & 0.0 \\
\hline Valine & $\mathrm{C} 00183$ & 1.1 & 0.1 & 0.3 & 1.1 & 0.1 & 0.2 & 0.5 & 0.1 & 0.0 \\
\hline Lactate & C00186 & 1.3 & 0.3 & 0.4 & 0.7 & 0.2 & 0.3 & 1.0 & 0.2 & 0.9 \\
\hline Threonine & $\mathrm{C} 00188$ & 0.8 & 0.1 & 0.0 & 0.9 & 0.1 & 0.1 & 0.5 & 0.1 & 0.0 \\
\hline $\begin{array}{l}\text { 2,3-Dihydroxybenzoic } \\
\text { acid }\end{array}$ & C00196 & 1.0 & 0.2 & 0.9 & 1.1 & 0.1 & 0.5 & 1.1 & 0.2 & 0.6 \\
\hline 3-phosphoglycerate & C00197 & 1.0 & 0.2 & 0.9 & 1.2 & 0.0 & 0.2 & 0.9 & 0.1 & 0.5 \\
\hline Adenosine & C00212 & 0.6 & 0.4 & 0.2 & 0.9 & 0.1 & 0.4 & 46.1 & 57.9 & 0.2 \\
\hline dCMP & C00239 & 0.6 & 0.2 & 0.3 & 0.6 & 0.2 & 0.3 & 3.2 & 2.1 & 0.1 \\
\hline Taurine & C00245 & 0.2 & 0.1 & 0.0 & 0.6 & 0.1 & 0.0 & 0.2 & 0.1 & 0.0 \\
\hline
\end{tabular}




\begin{tabular}{|c|c|c|c|c|c|c|c|c|c|c|}
\hline Pyridoxal & $\mathrm{C} 00250$ & 1.3 & 0.4 & 0.3 & 1.0 & 0.1 & 0.9 & 1.0 & 0.2 & 0.9 \\
\hline Riboflavin & $\mathrm{C} 00255$ & 1.4 & 0.5 & 0.4 & 0.9 & 0.2 & 0.9 & 1.2 & 0.3 & 0.6 \\
\hline Carnitine & $\mathrm{C} 00318$ & 1.7 & 0.4 & 0.1 & 1.0 & 0.2 & 0.9 & 0.8 & 0.3 & 0.3 \\
\hline dGDP & $\mathrm{C} 00361$ & 0.6 & 0.1 & 0.1 & 0.9 & 0.1 & 0.8 & 0.3 & 0.2 & 0.0 \\
\hline Xanthine & $\mathrm{C} 00385$ & 1.3 & 0.1 & 0.0 & 1.1 & 0.1 & 0.2 & 1.7 & 0.2 & 0.0 \\
\hline Acetyl-glutamic acid & $\mathrm{C} 00624$ & 0.8 & 0.1 & 0.1 & 0.9 & 0.1 & 0.3 & 0.6 & 0.1 & 0.0 \\
\hline Creatinine & C00791 & 1.3 & 0.3 & 0.4 & 0.8 & 0.2 & 0.5 & 0.9 & 0.2 & 0.7 \\
\hline Pantothenic Acid & $\mathrm{C} 00864$ & 0.7 & 0.1 & 0.0 & 1.0 & 0.1 & 0.6 & 0.7 & 0.1 & 0.0 \\
\hline L-cystathionine & C02291 & 0.6 & 0.1 & 0.0 & 0.8 & 0.1 & 0.0 & 0.3 & 0.1 & 0.0 \\
\hline $\begin{array}{c}\text { 4-Amino-3- } \\
\text { hydroxybutyrate }\end{array}$ & C03678 & 0.5 & 0.2 & 0.0 & 0.8 & 0.1 & 0.0 & 0.5 & 0.1 & 0.0 \\
\hline Arachidic Acid & C06425 & 1.1 & 0.1 & 0.4 & 1.0 & 0.1 & 0.7 & 1.2 & 0.2 & 0.2 \\
\hline Eicosatrienoic acid & C16522 & 0.8 & 0.1 & 0.1 & 0.8 & 0.2 & 0.2 & 0.8 & 0.3 & 0.2 \\
\hline Eicosadienoic acid & C16525 & 1.1 & 0.2 & 0.4 & 1.1 & 0.2 & 0.6 & 0.9 & 0.1 & 0.4 \\
\hline Eicosenoic acid & C16526 & 1.0 & 0.3 & 0.8 & 1.0 & 0.2 & 0.8 & 1.1 & 0.2 & 0.5 \\
\hline $\begin{array}{l}\text { Beta-Citryl-L- } \\
\text { glutamic acid }\end{array}$ & C20775 & 0.7 & 0.1 & 0.0 & 0.7 & 0.1 & 0.0 & 0.4 & 0.2 & 0.0 \\
\hline $\begin{array}{c}6- \\
\text { Dimethylaminopurine }\end{array}$ & HMDB0000473 & 1.1 & 0.2 & 0.2 & 1.0 & 0.1 & 0.5 & 52.0 & 67.6 & 0.2 \\
\hline Octadecanedioic acid & HMDB0000782 & 1.1 & 0.3 & 0.7 & 1.1 & 0.4 & 0.6 & 2.3 & 1.5 & 0.2 \\
\hline $\begin{array}{l}\text { CMP-N- } \\
\text { acetylneuraminic acid }\end{array}$ & HMDB0001176 & 0.5 & 0.1 & 0.0 & 0.9 & 0.1 & 0.3 & 0.3 & 0.2 & 0.0 \\
\hline 3-Methyluric acid & HMDB0001970 & 0.8 & 0.1 & 0.1 & 0.8 & 0.2 & 0.2 & 0.6 & 0.1 & 0.0 \\
\hline
\end{tabular}


158 Table S8. Joint pathway analysis for integration of metabolomics and transcriptomics data

\begin{tabular}{|c|c|c|c|c|c|c|c|c|c|}
\hline Pathways & Total & Expected & Hits & $\begin{array}{c}\text { Raw } \\
p\end{array}$ & $-\log (p)$ & $\begin{array}{c}\text { Holm } \\
\text { adjust }\end{array}$ & FDR & Impact & $\begin{array}{c}\text { Fold } \\
\text { Enrichment }\end{array}$ \\
\hline $\begin{array}{c}\text { Aminoacyl-tRNA } \\
\text { biosynthesis }\end{array}$ & 87 & 3.39 & 10 & 0.001 & 6.6 & 0.1089 & 0.11 & 0.20 & 3.0 \\
\hline $\begin{array}{c}\text { Steroid } \\
\text { biosynthesis }\end{array}$ & 54 & 2.10 & 6 & 0.016 & 4.1 & 1 & 0.51 & 0.65 & 2.9 \\
\hline $\begin{array}{c}\text { Alanine,aspartate } \\
\text { and glutamate } \\
\text { metabolism }\end{array}$ & 56 & 2.18 & 6 & 0.019 & 4.0 & 1 & 0.51 & 0.63 & 2.8 \\
\hline $\begin{array}{c}\text { D-Glutamine and } \\
\text { D-glutamate } \\
\text { metabolism }\end{array}$ & 9 & 0.35 & 2 & 0.045 & 3.1 & 1 & 0.90 & 0.57 & 5.7 \\
\hline
\end{tabular}

159

160 
EC $_{50}$ calculation of Chemical A and Chemical B

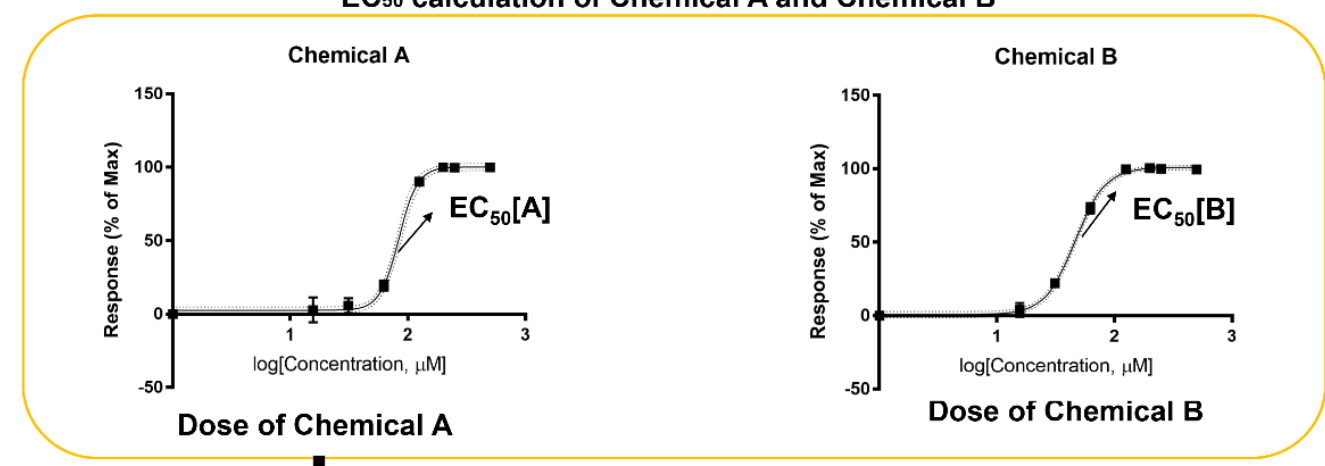

Dual Chemicals Treatment: Chemical A and Chemical B were further mixed with a fixed ratio of $\mathrm{m} / \mathrm{n}$ (' $1: 4$ ', ' $2: 3$ ', ' $3: 2$ ' or ' $4: 1$ ' in this study)

$\mathrm{EC}_{50}[\mathrm{~A}+\mathrm{B}]$ calculation for dual chemicals treatment

161

162

163

164

165

166

167

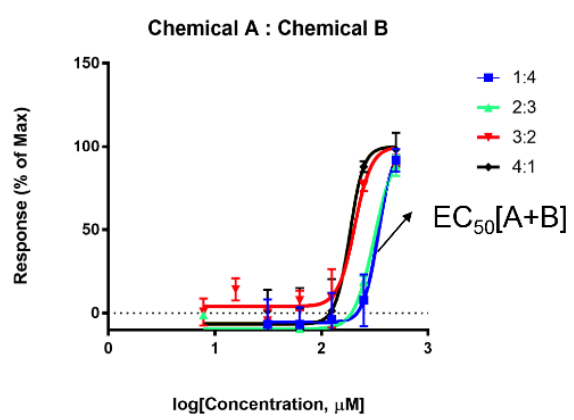

Figure S1. The determination and calculation steps of combination index (CI) of 78 dual chemical combinations in the chemical interaction screening test (Use $\mathrm{EC}_{50}$ as an example; the mixing ratio for dual chemicals were $\mathrm{m} / \mathrm{n}=={ }^{\prime} 1: 4$ ', ' $2: 3$ ', ' $3: 2$ ' or ' $4: 1$ ' in this study; at least 6 times' serial dilution were prepared using growth medium with a diluting factor of 2 ; the nominal maximal combined dosing concentration is $500 \mu \mathrm{M}$ for each pair) 
A

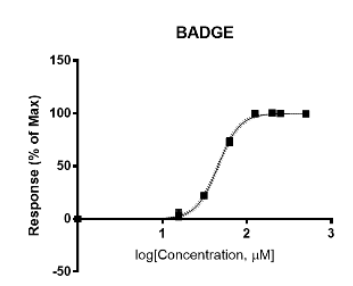

E

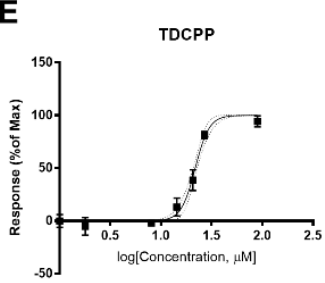

I

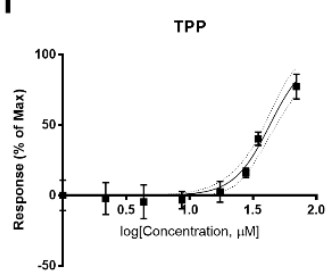

168
B

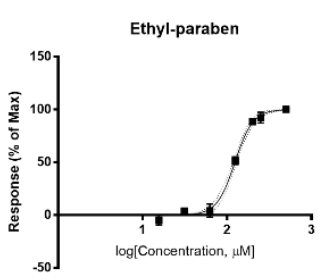

F
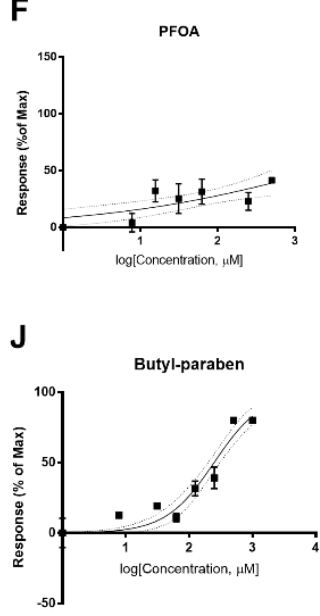

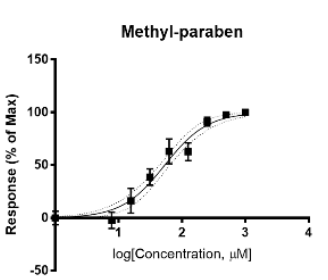

G

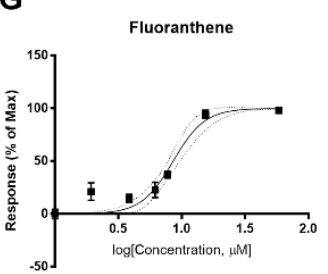

K

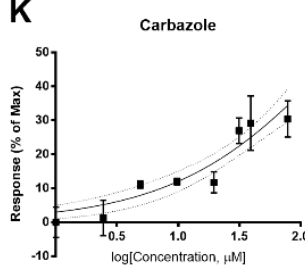

D

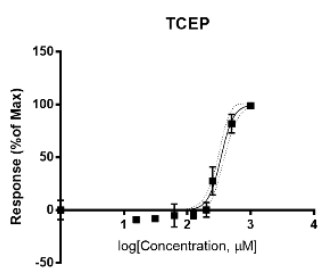

H BPA

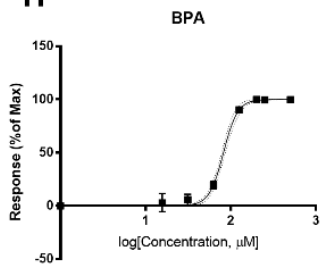

L B[a]p

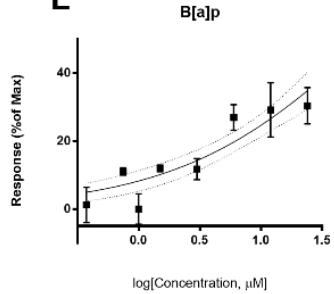

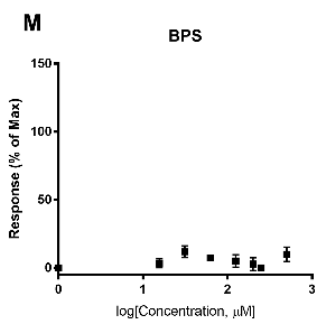

170 Figure S2. A to M. Dose-Response curve of 13 selected chemicals fitted using "log[dose]-response-

171 inhibition-viable slope: four parameters" model; $\mathrm{x}$-axis represents $\log$ [concentration, $\mu \mathrm{M}$ ] and $\mathrm{y}$-axis 172 represents normalized response (\% of maximal response); dash line represents the $95 \%$ confidence 173 interval band; note that carbazole and $\mathrm{B}[\mathrm{a}] \mathrm{p}$ did not reach $50 \%$ inhibition so their $\mathrm{CI}$ values were based 174 on their $\mathrm{EC}_{25}$ values. For single chemical did not exhibit apparent cytotoxicity effect such as BPS and 175 PFOA, their a/A portion was approximated to 0 for calculation. 

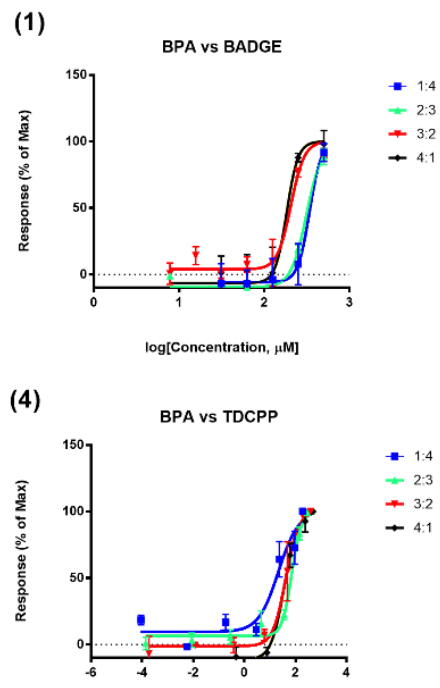

176
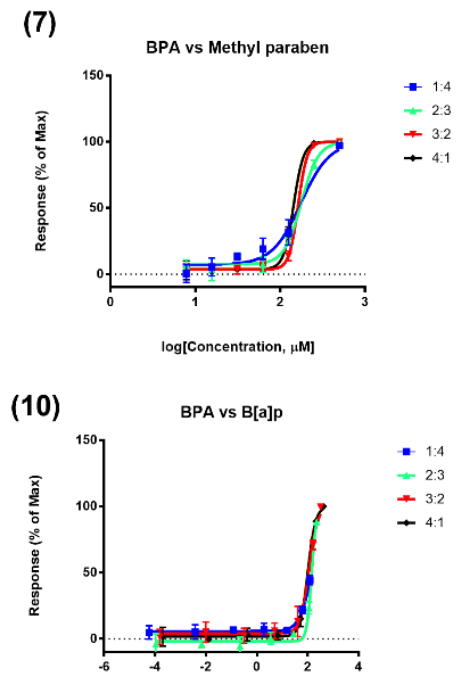

log[Concentration, $\mu M]$

(13)

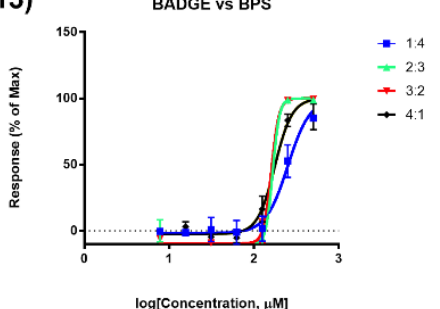

(2)

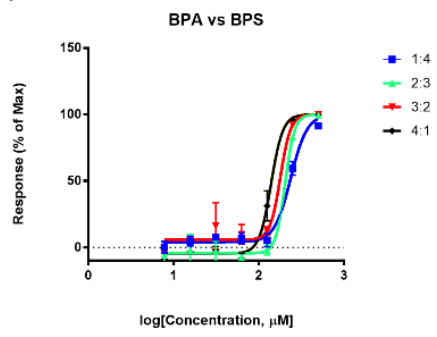

(5)

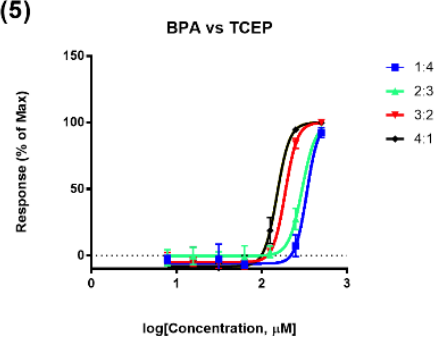

(8)

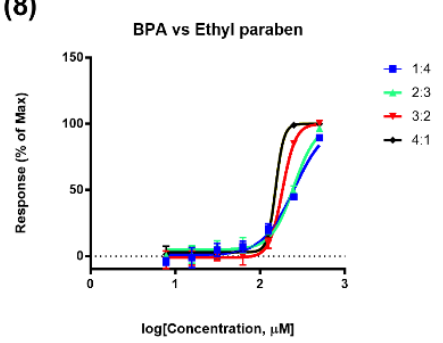

(11)

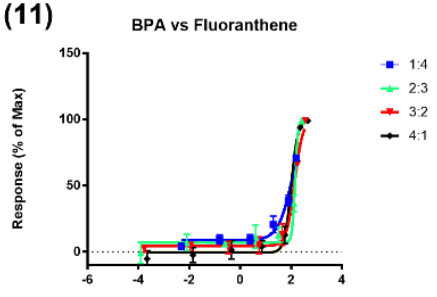

(14)

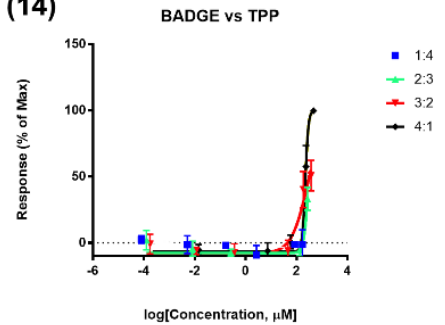

(3)

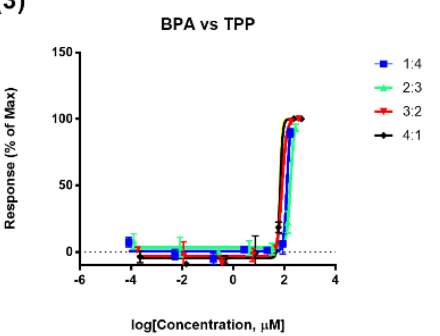

(6)

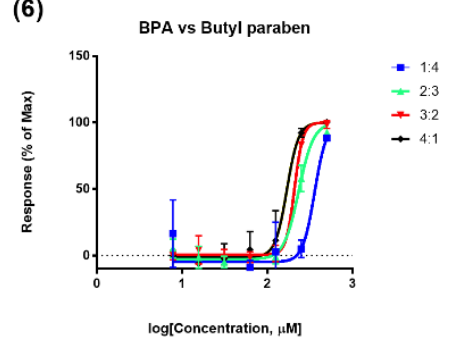

(9)

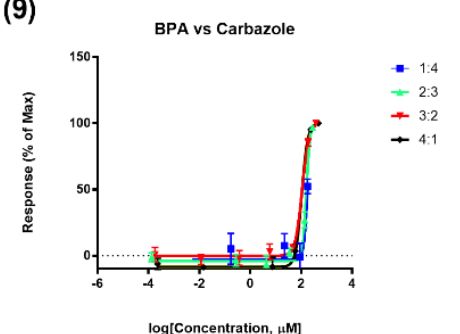

(12)
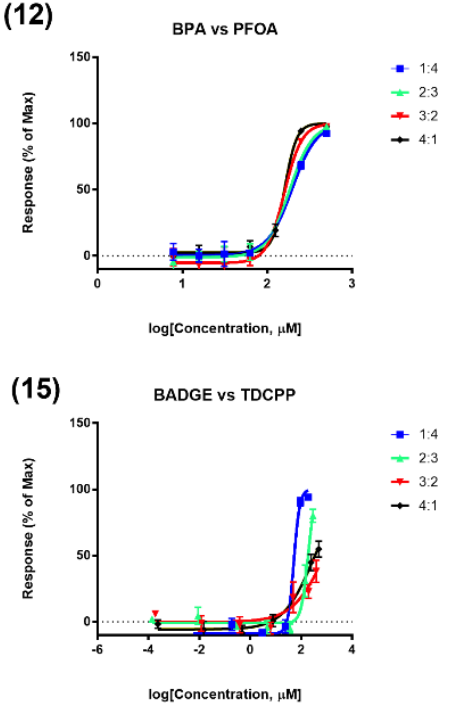

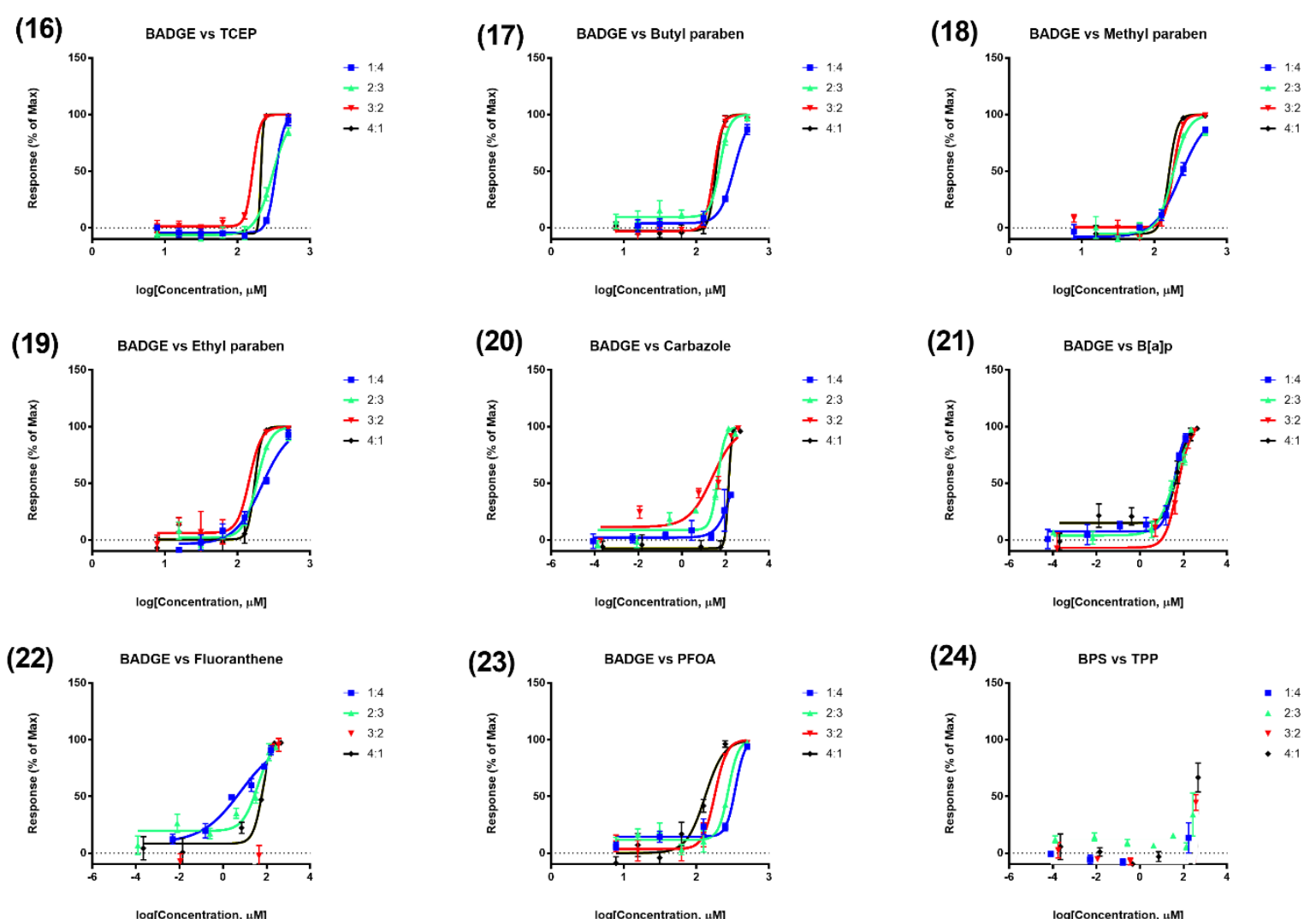

178
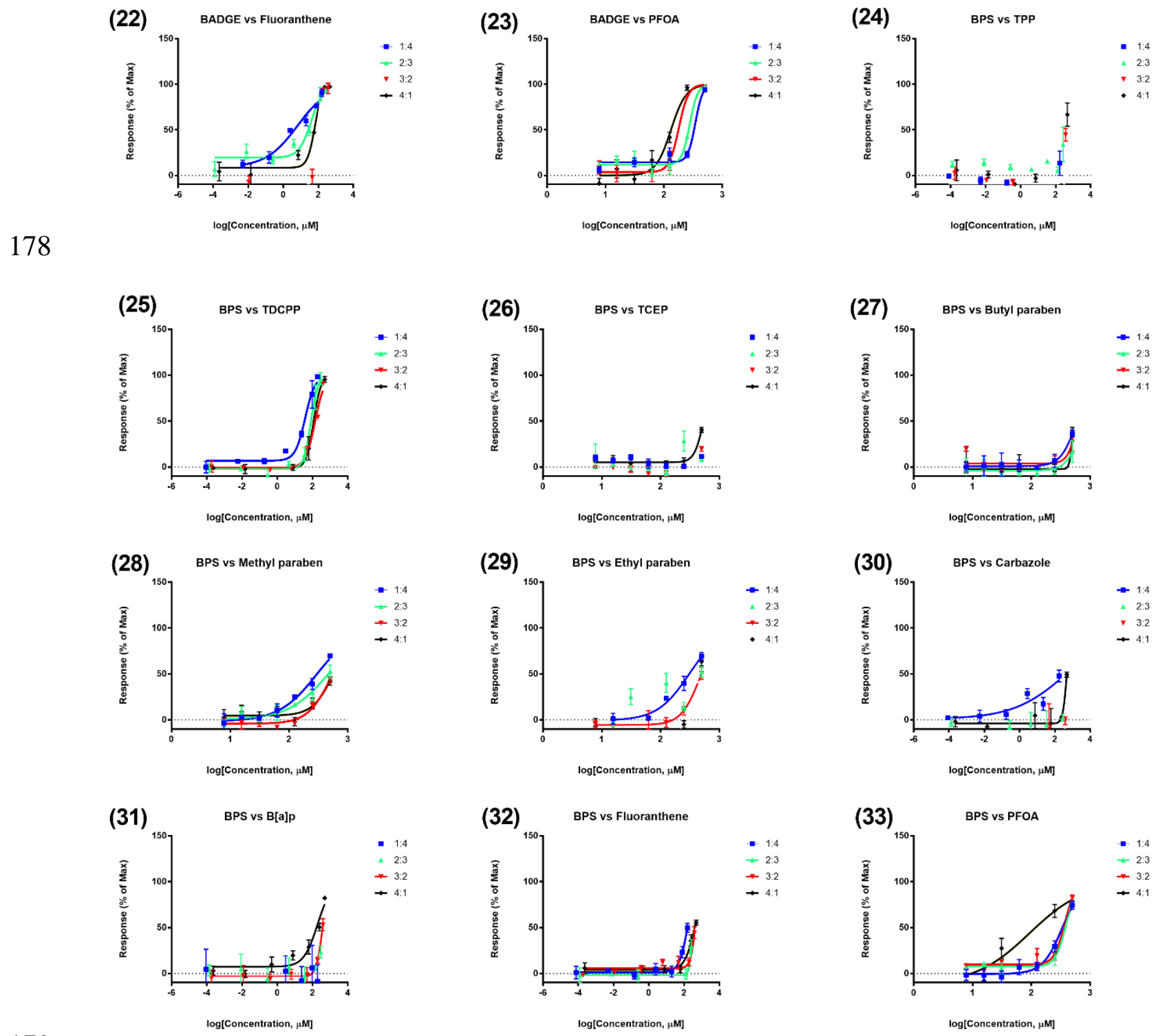

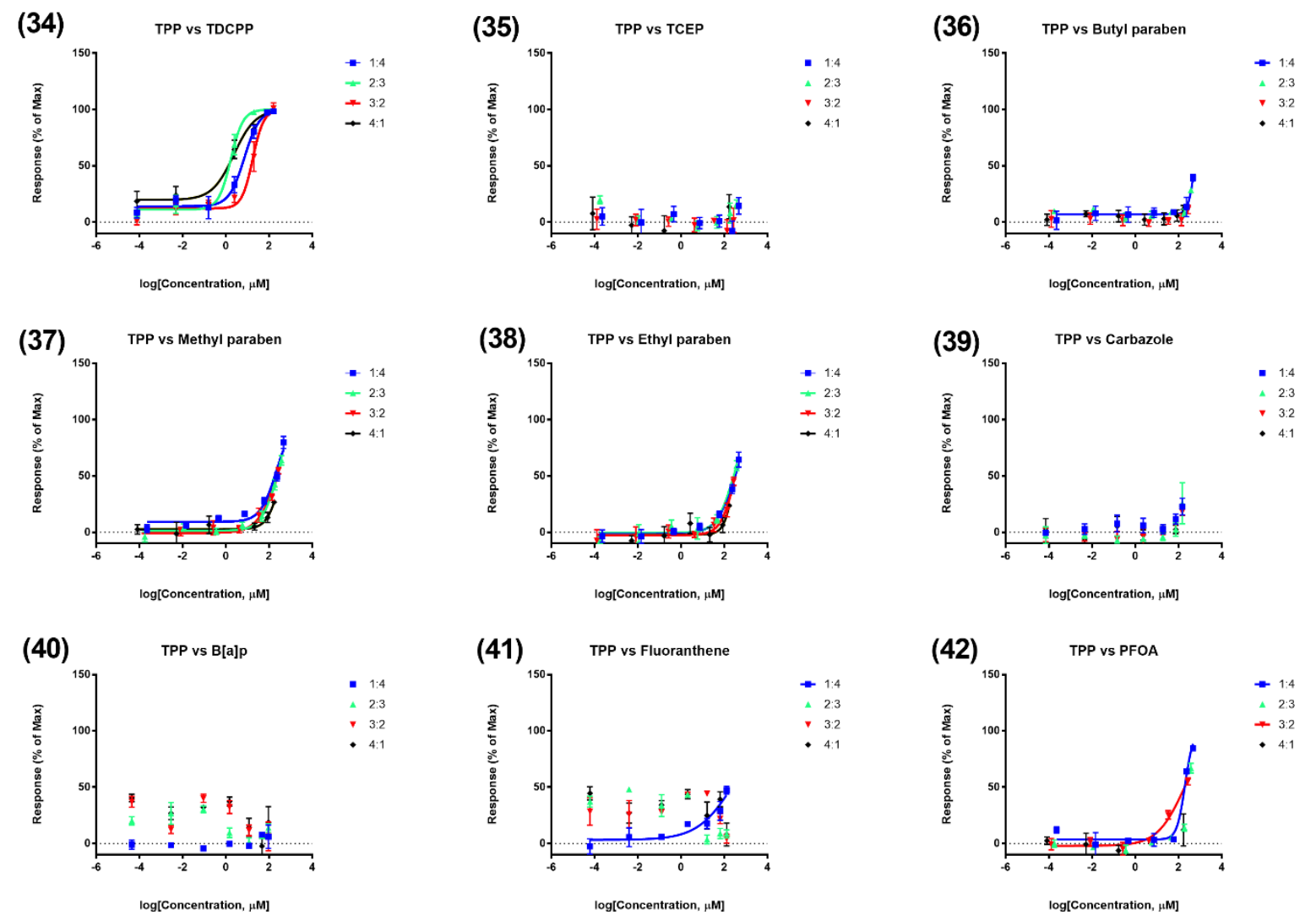

180
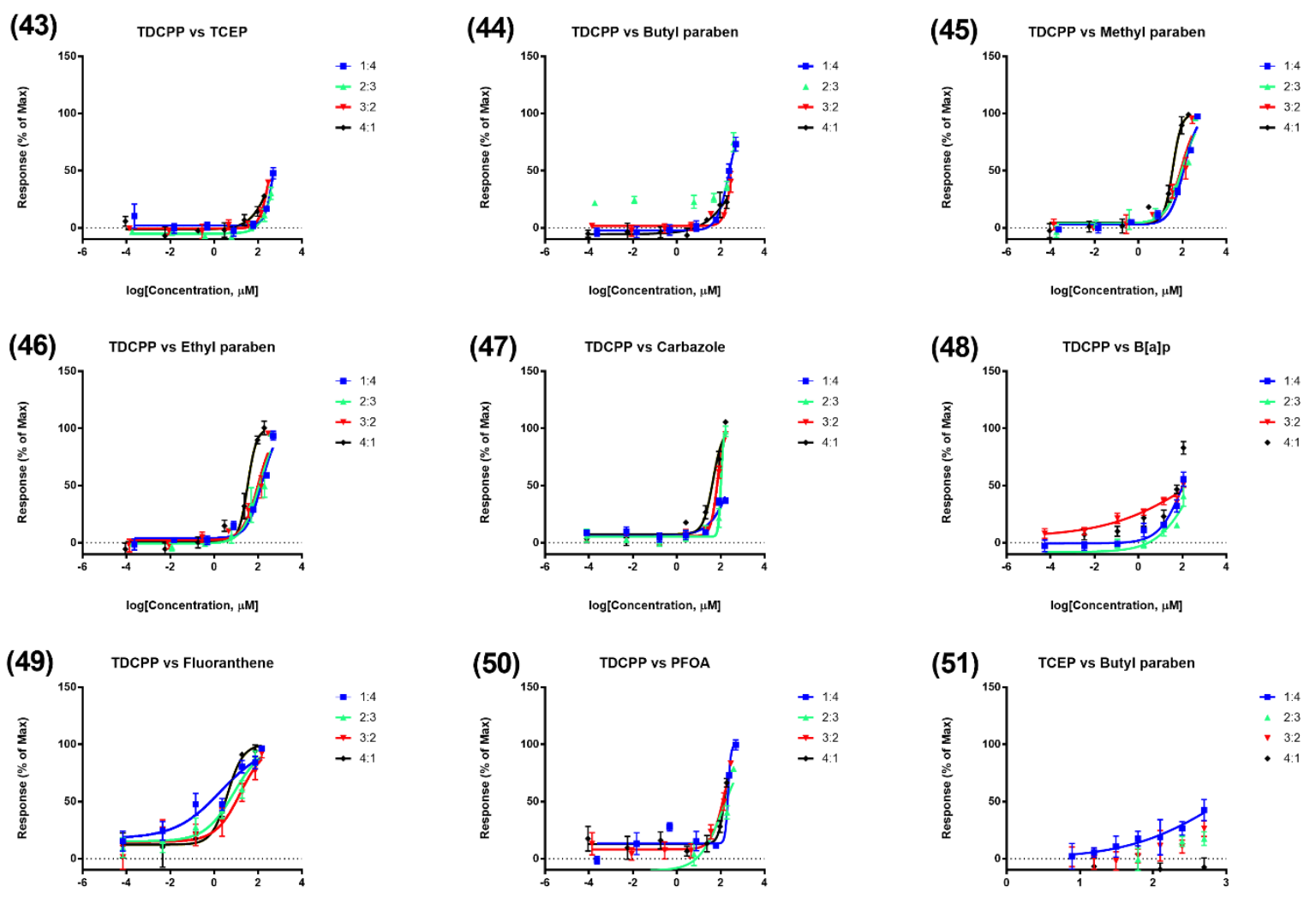

181

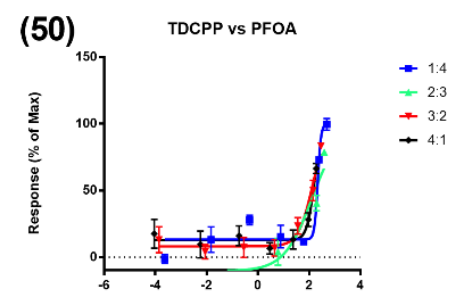

log[Concentration, $\mu \mathrm{M}$ ]

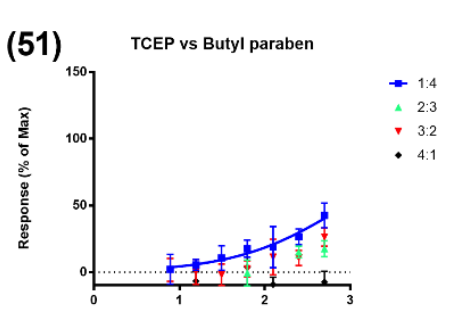

log[Concentration, $\mu \mathrm{M}]$ 
(52)

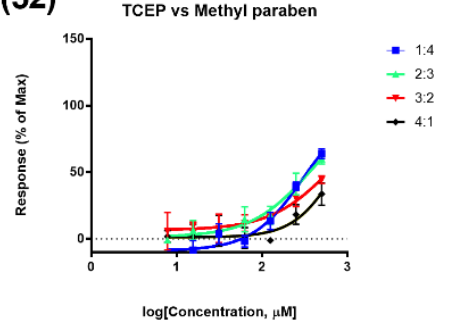

(55)

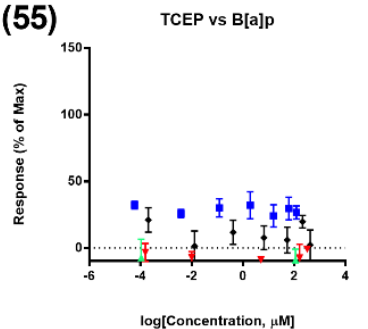

(58)

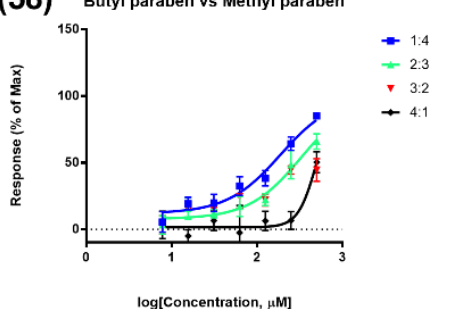

182

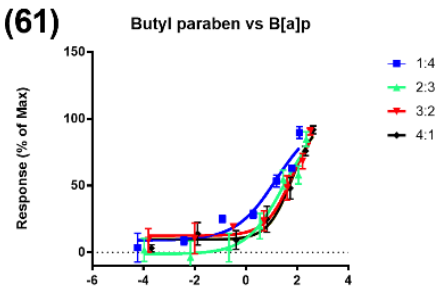

(64) Methyl paraben vs Ethyl paraben

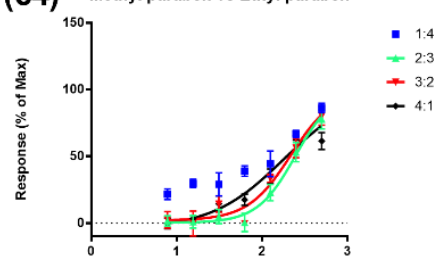

log[Concentration, $\mu \mathrm{M}]$

(67) Methyl paraben vs Fluoranthene

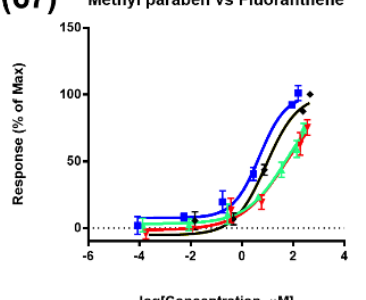

183

184
(53)

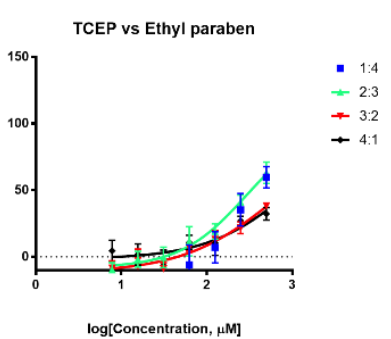

(56)

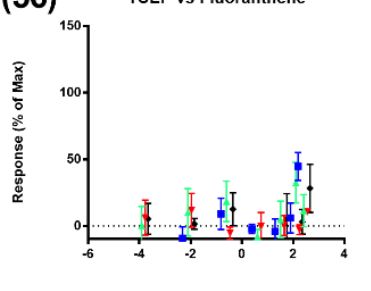

(59) Butyl paraben vs Ethyl paraben

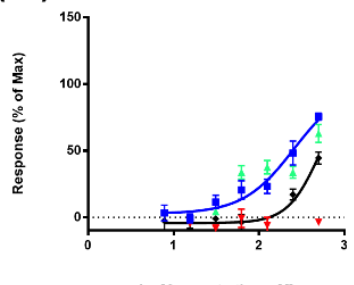

log[Concentration, $\mu \mathrm{MM}]$

(62)

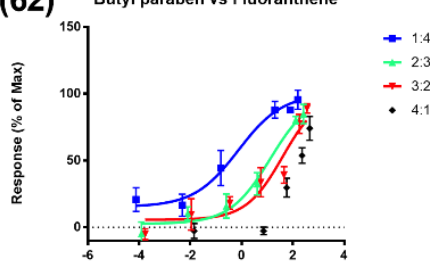

log[Concentration, $\mu \mathrm{M}]$

(65) Methyl paraben vs Carbazole

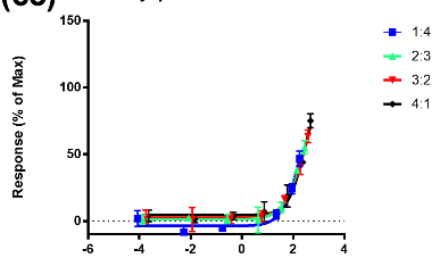

log[Concentration, $\mu \mathrm{M}]$

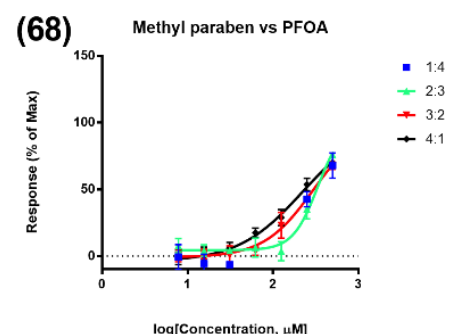

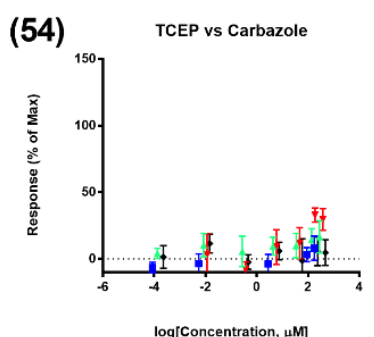

(57)

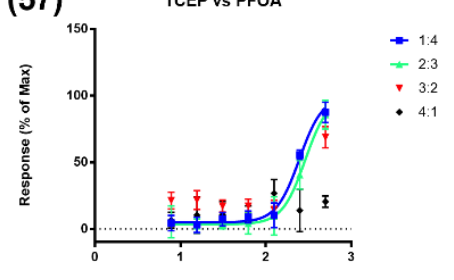

(60) Buyty paraben vs Carbazole

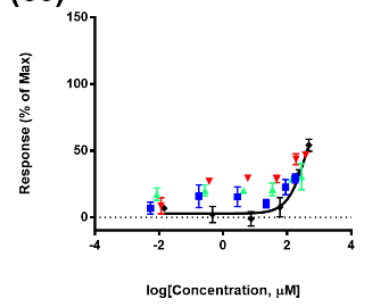

(63) Butyl paraben vs PFOA
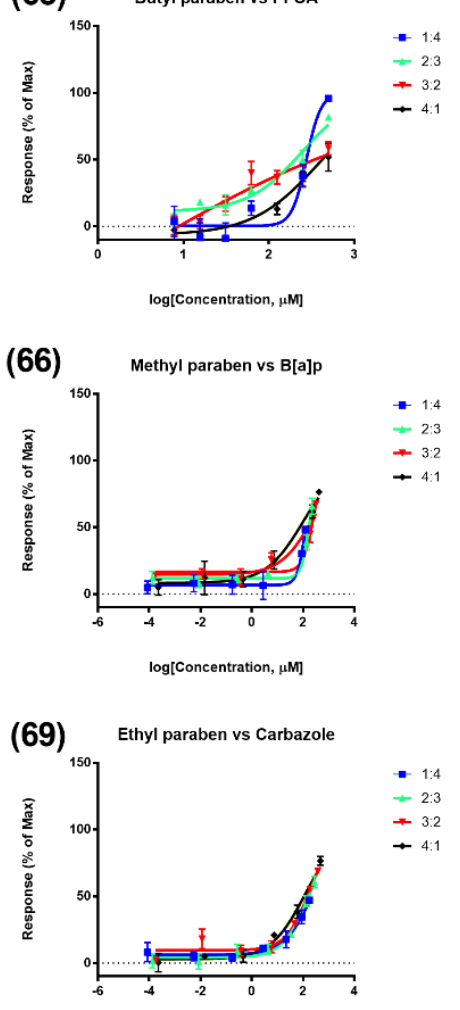

log[Concentration, $\mu \mathrm{M}]$ 


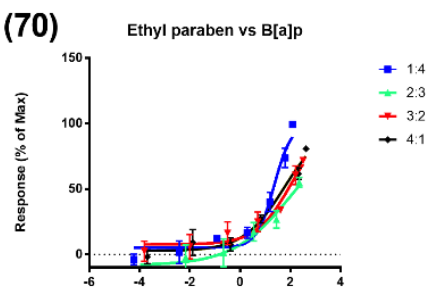

log[Concentration, $\mu \mathrm{M}]$

(73)

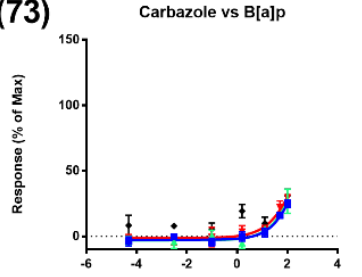

log[Concentration, $\mu M]$

(76)

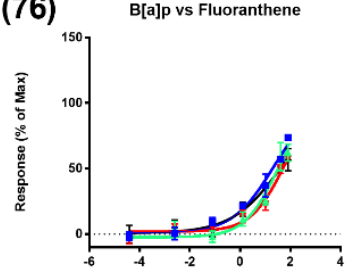

log[Concentration, $\mu \mathrm{M}]$

$$
\begin{array}{r}
-1: 4 \\
+2.3 \\
-3.2 \\
\hline 4.1
\end{array}
$$

(71)

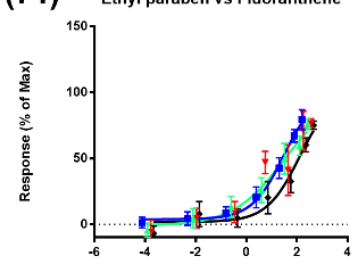

log[Concentration, $\mu \mathrm{M}]$

(74)

185

186

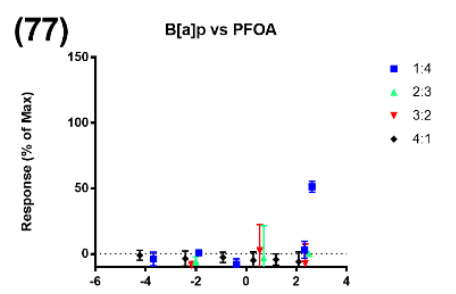

log[Concentration, $\mu \mathrm{M}]$
(2)

(9)

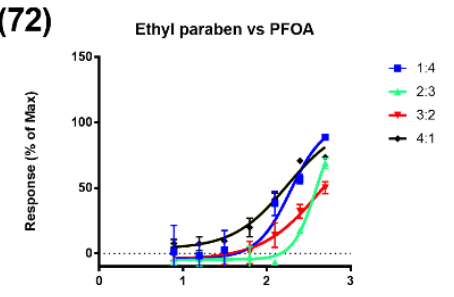

log[Concentration, $\mu \mathrm{M}]$

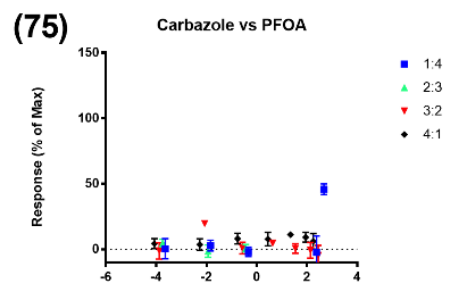

$\log [$ Concentration, $\mu \mathrm{M}]$
log[Concentration, $\mu \mathrm{M}]$

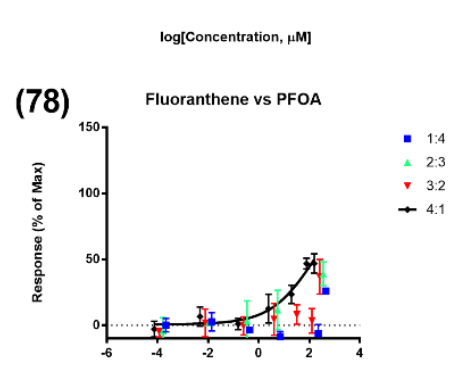

Figure S3. (1) to (78). Dose-Response curve of 78 dual chemicals combinations with different mixing ratio (' $1: 4$ ', ' $2: 3$ ', ' $3: 2$ ' or ' $4: 1$ ' ) fitted using " $\log [\operatorname{dose}]$-response-inhibition-viable slope: four parameters" model; $\mathrm{x}$-axis represents $\log [$ concentration, $\mu \mathrm{M}]$ and $\mathrm{y}$-axis represents normalized response (\% of maximal response); curve will be exempted for fitting if a. not follow sigmoidal dose-response relationship; b. no significant response was observed or c. curve bottom is way above/below 0 . 

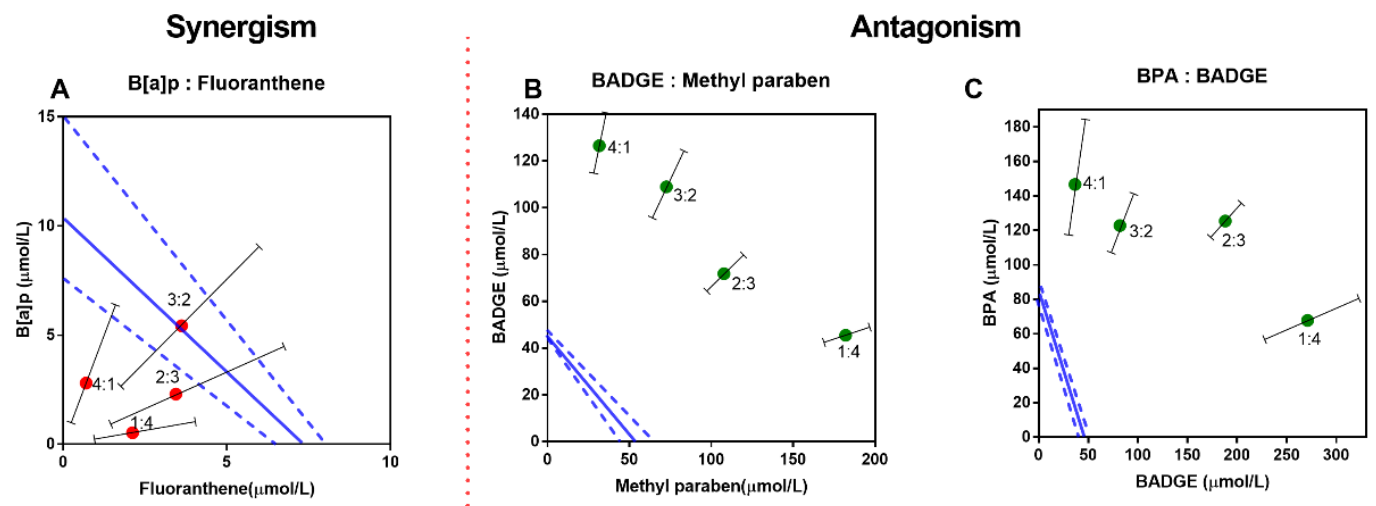

Additive Effect

D

$\mathrm{E}$
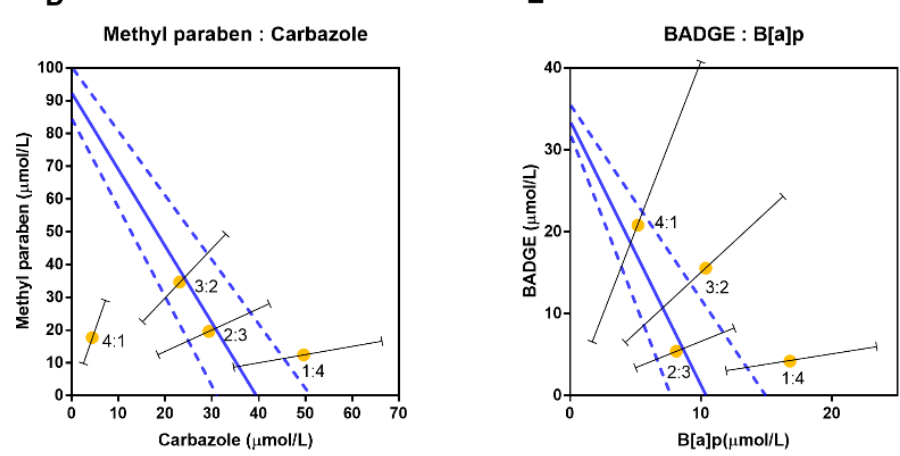

197 Figure S4. Isobolograms of selected chemical pairs ( ' $1: 4{ }^{\prime}$, ' '2:3' , '3:2' and '4:1' )

198 for the inhibition on cell growth of HepG2. A. B[a]p and fluoranthene at $\mathrm{EC}_{25} ; \mathbf{B}$. BADGE and 199 methyl paraben at $\mathrm{EC}_{50} ; \mathbf{C}$. BPA and $\mathrm{BADGE}$ at $\mathrm{EC}_{50} ; \mathbf{D}$. Methyl paraben and carbazole at $200 \mathrm{EC}_{25}$ and $\mathbf{E}$. BADGE and $\mathrm{B}[\mathrm{a}] \mathrm{p}$ at $\mathrm{EC}_{25}(\vdash \bullet-1$ represents effect concentration values with $95 \%$ 201 confidence interval for the combination ratios indicated. $\equiv \equiv \equiv$ represents additivity line with $20295 \%$ confidence belt, the $\mathrm{x}$-axis and y-axis represent the concentrations $(\mu \mathrm{mol} / \mathrm{L})$ of two 203 chemicals. The area between the two diagonal dotted lines in each plot represents the additive effect; the triangular area under the additive effect region represents the synergistic effect; the area above the additive effect area represent antagonist effect. 


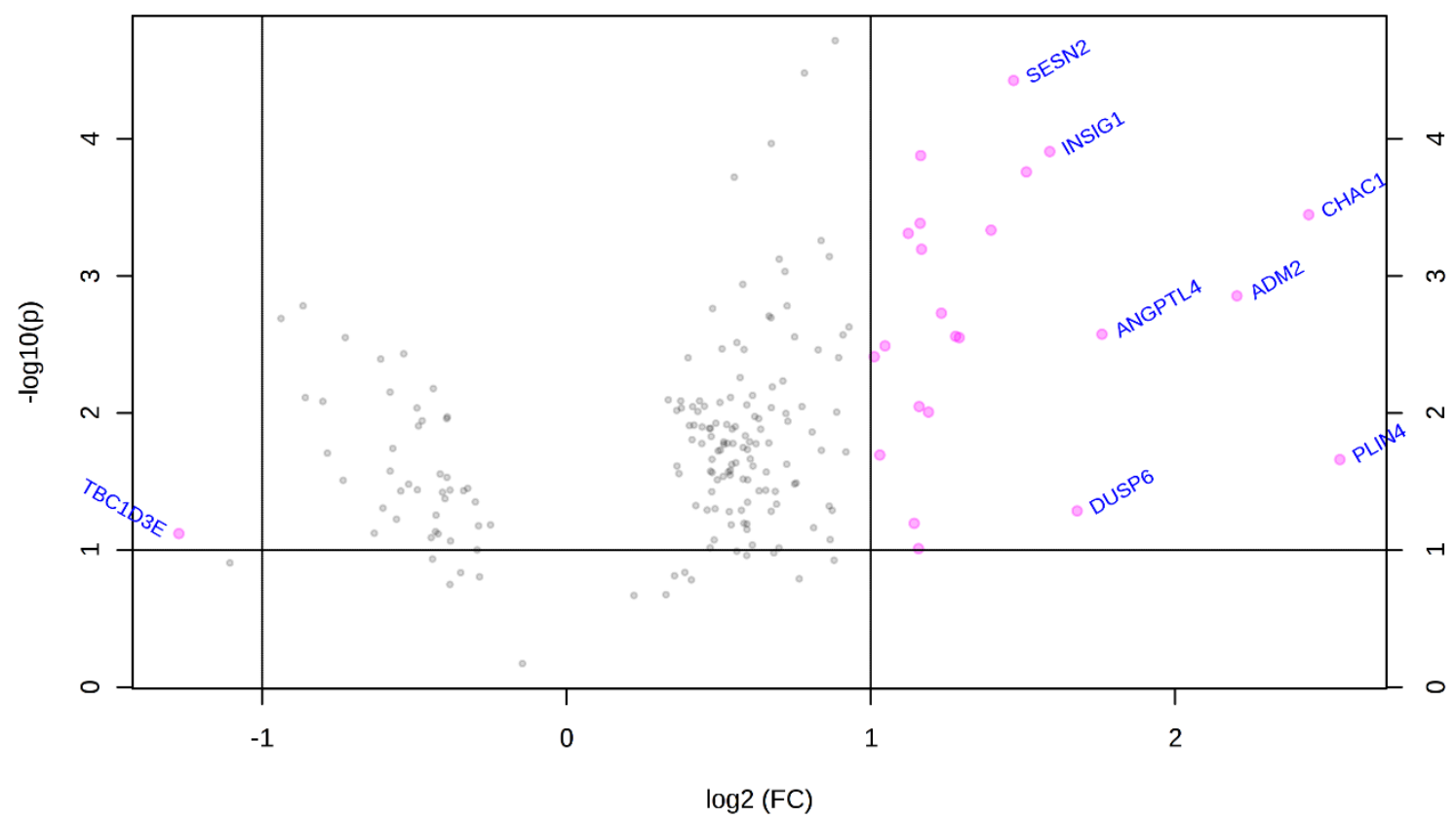

Figure S5. Volcano plot illustrating differential expression of genes in Dual versus Control samples. 


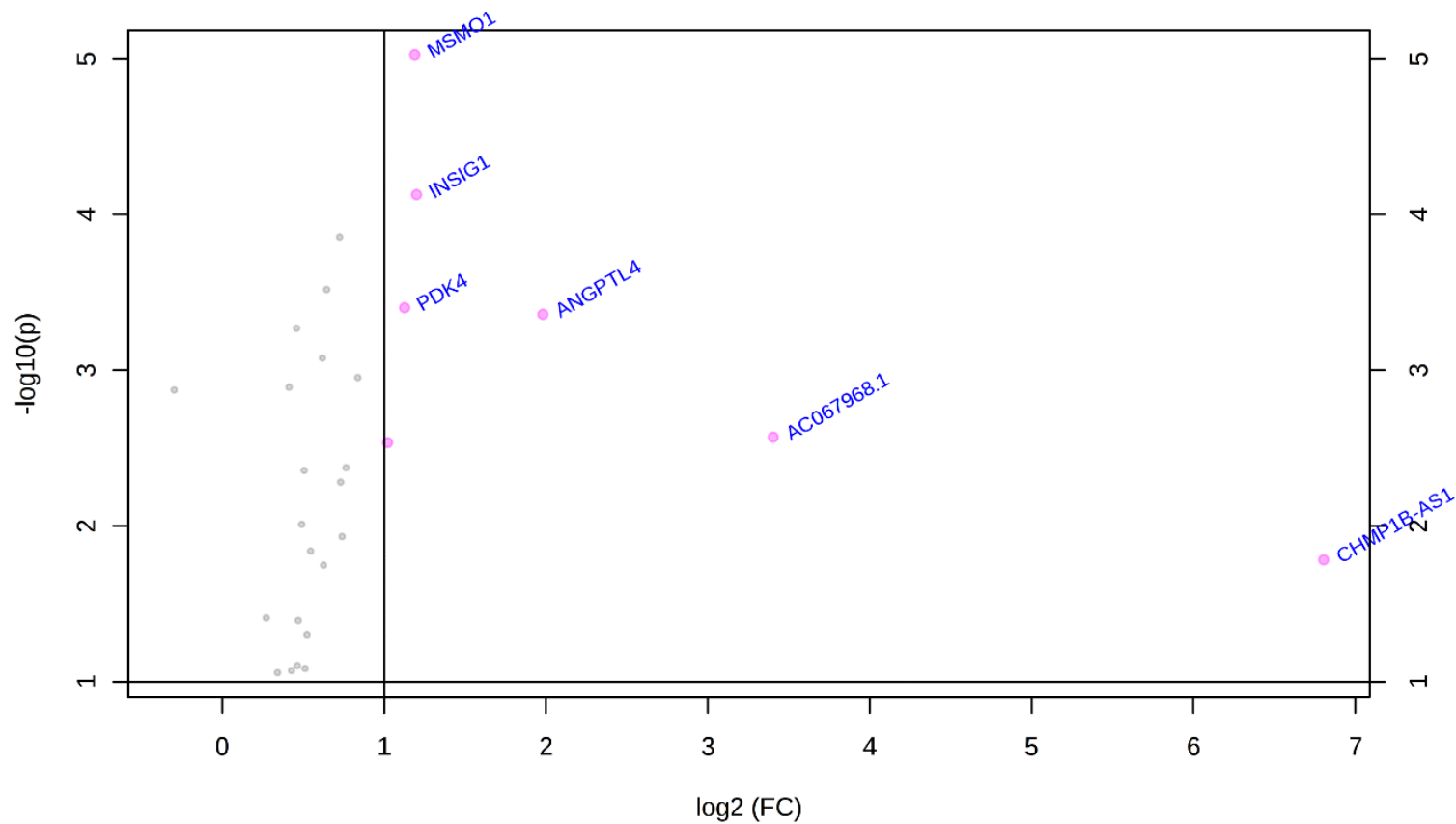

210

211 Figure S6. Volcano plot illustrating differential expression of genes in TDCPP versus Control samples. 


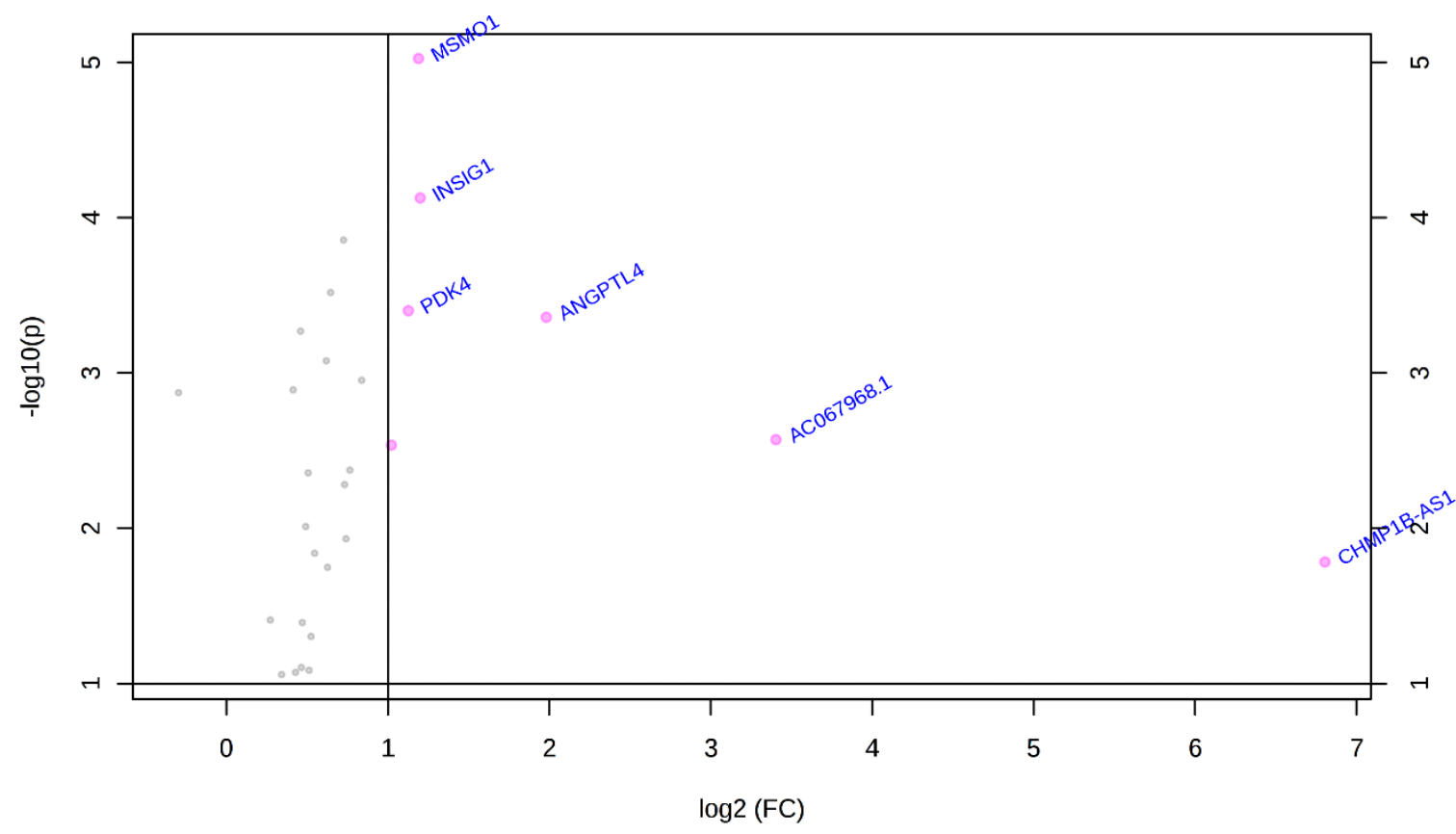

215 Figure S7. Volcano plot illustrating differential expression of genes in TPP versus Control samples. 


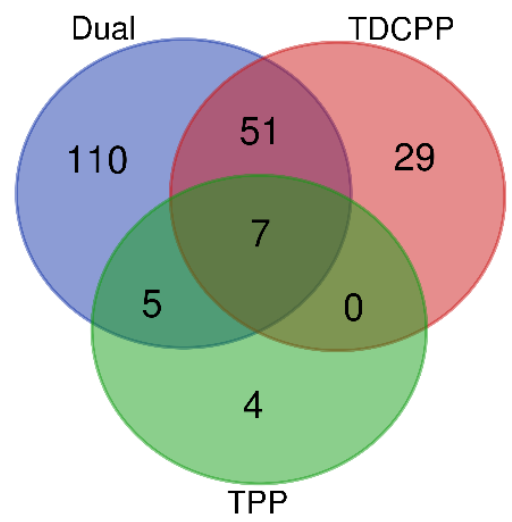

Figure S8. Classic Venn diagram summarizing the number of shared and distinct GO terms for three treatment conditions. 
A. Dual vs Ctrl

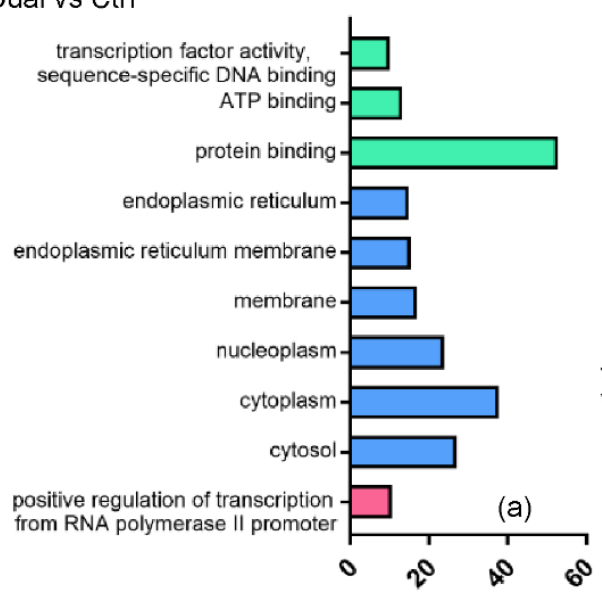

C. TPP vs Ctrl

Percentage of genes $(\%)$

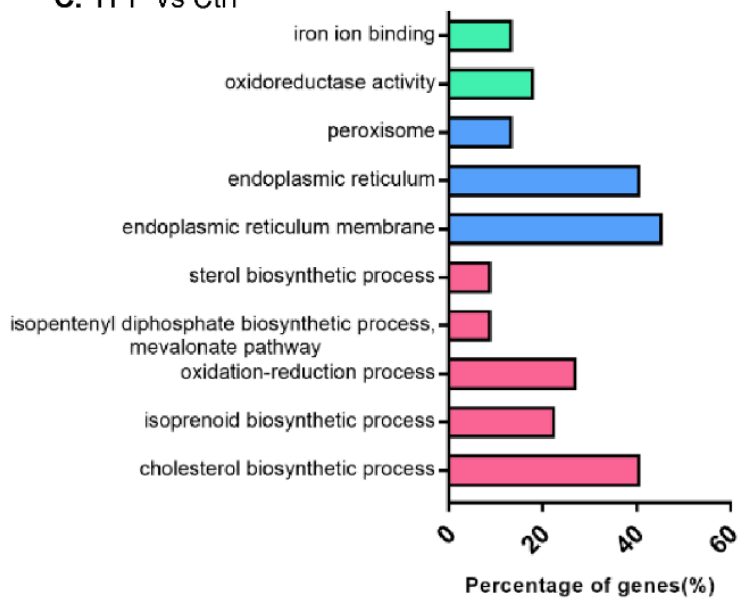

B. TDCPP vs Ctrl

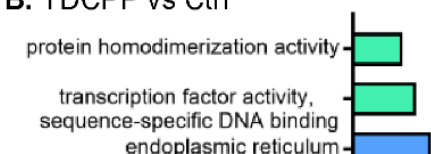

Biological Process

$\square$ Cellular Component

$\square$ Molecular Function

endoplasmic reticulum membrane

cytoplasm

regulation of transcription from

RNA polymerase II promoter intrinsic apoptotic signaling pathway in esponse to endoplasmic reticulum stress esponse to endoplasmic reticulum stress

negative regulation of transcription from RNA polymerase II promoter

positive regulation of transcription

from RNA polymerase II promoter

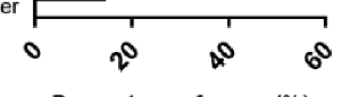

D. All overlapping genes

Percentage of genes $(\%)$

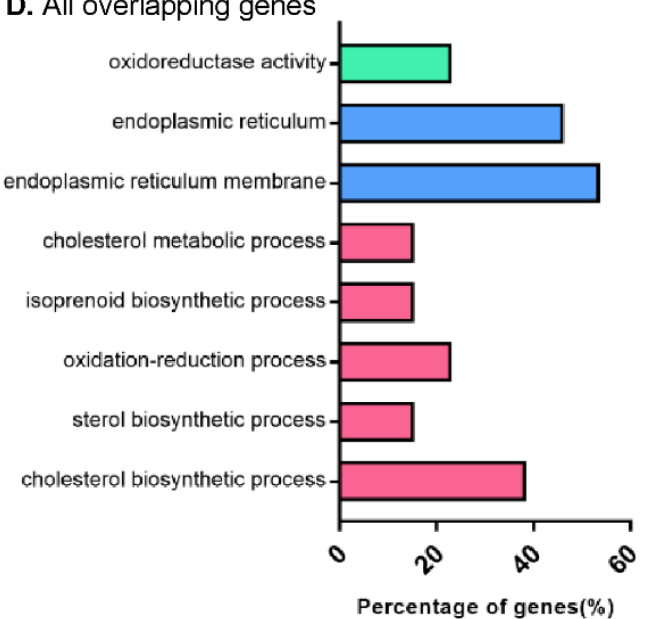

226 Figure S9. Gene Ontology (GO) analysis results from DAVID Bioinformatics Resources 6.8 for each

227 treatment conditions A. Dual vs Control; B. TDCPP vs Control; C. TPP vs Control; D. overlapped genes for all treatment conditions 
A

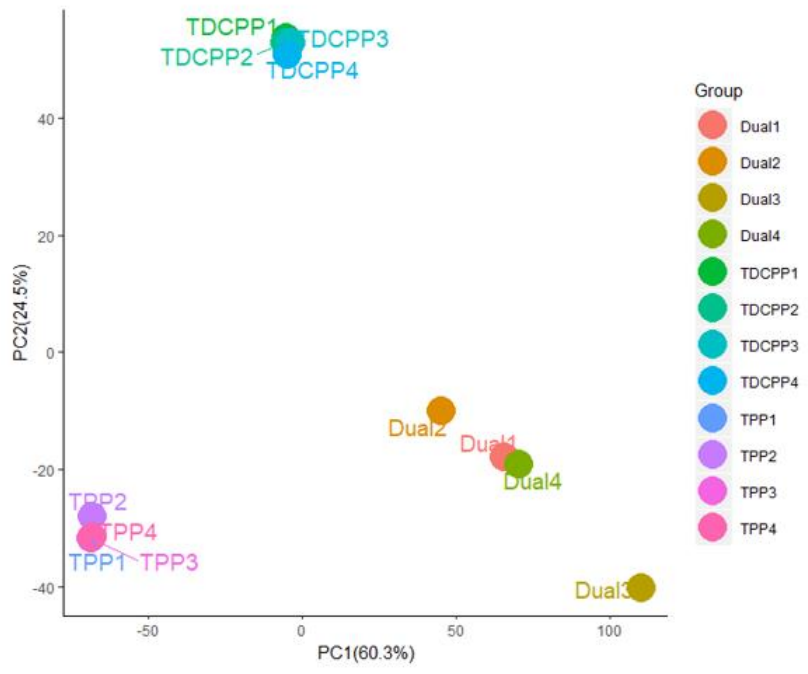

B

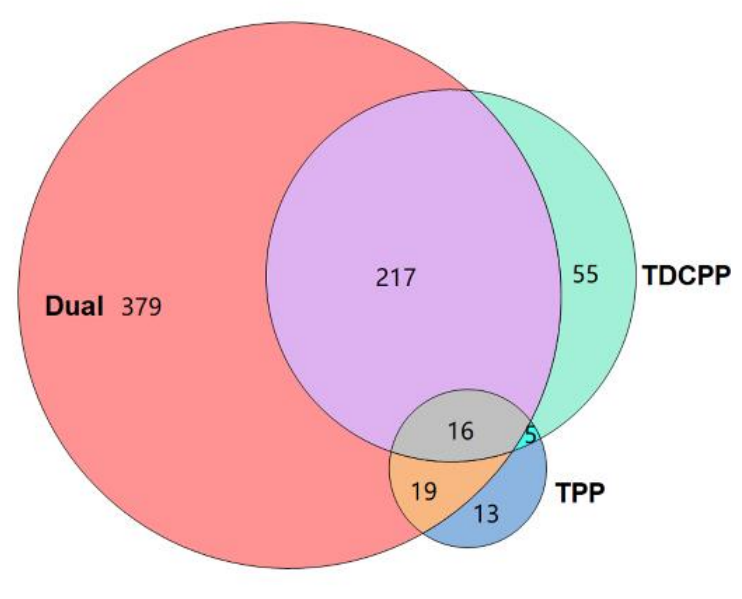

248 Figure S10. Analysis of dysregulated features in response to TDCPP, TPP and Dual exposure.

249 A. Principal component analysis (PCA) of the dysregulated features for different groups. B.

250 Classic Venn diagram summarizing the number of shared and distinct dysregulated metabolite 251 features for three treatment conditions. 


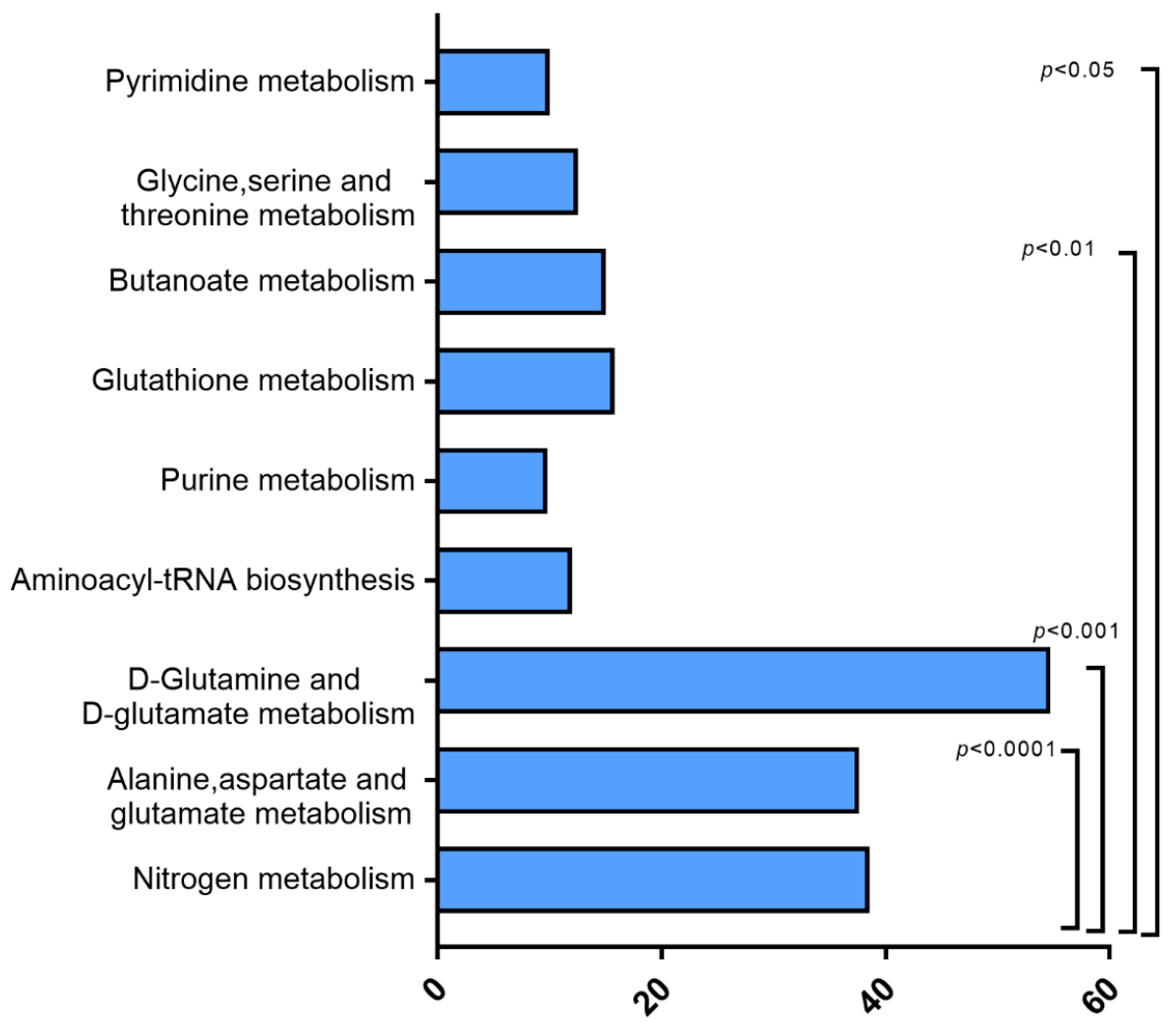

Fold Enrichment

256 Figure S11. Enrichment analysis of significant metabolic pathways of HepG2 treated with TPP. The data analysis was conducted by the open source platform MetaboAnalyst. 


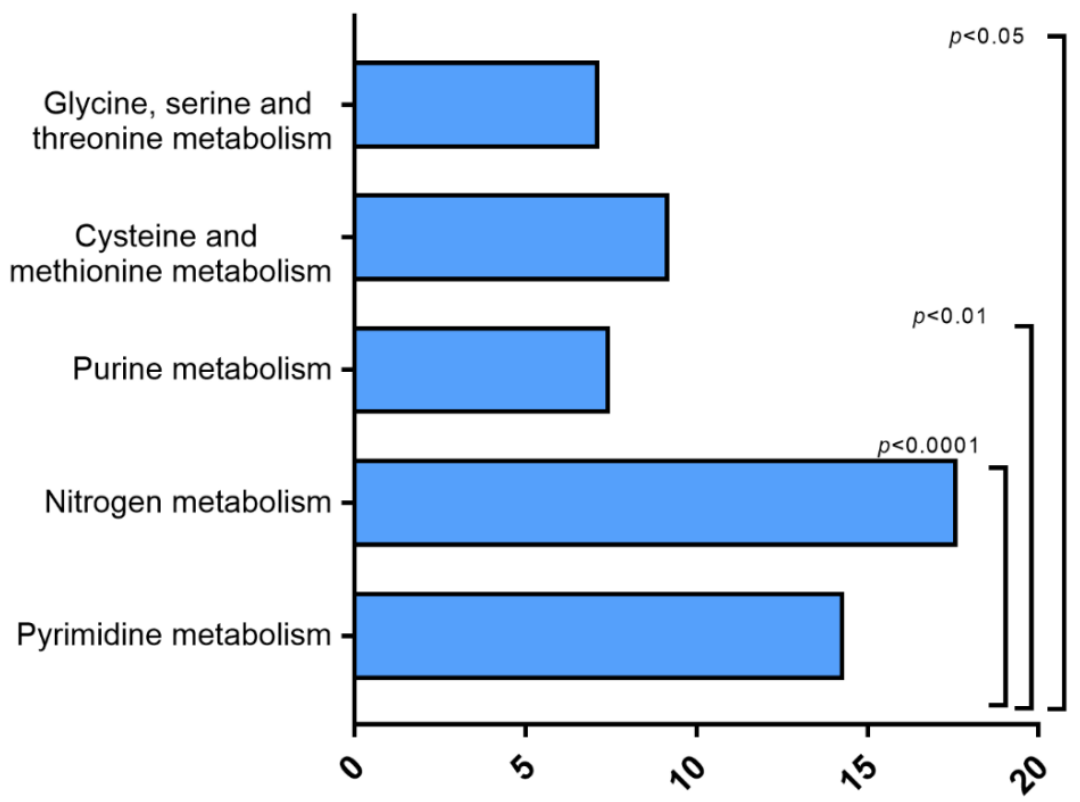

Fold Enrichment

261 Figure S12. Enrichment analysis of significant metabolic pathways of HepG2 treated with TDCPP. The data analysis was conducted by the open source platform MetaboAnalyst. 


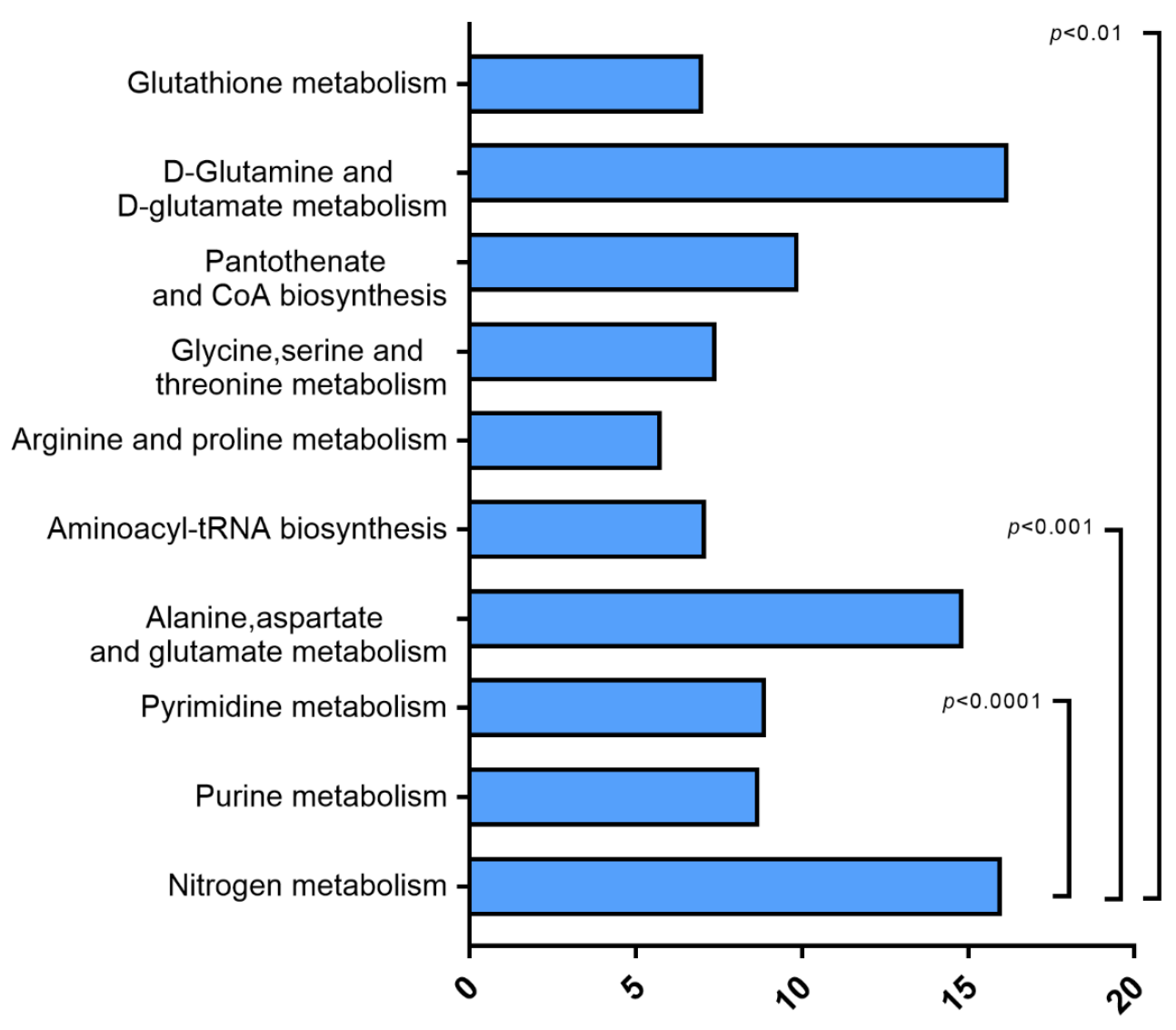

Fold Enrichment

Figure S13. Enrichment analysis of significant metabolic pathways of HepG2 treated with Dual. The 
(a) Purine Metabolism

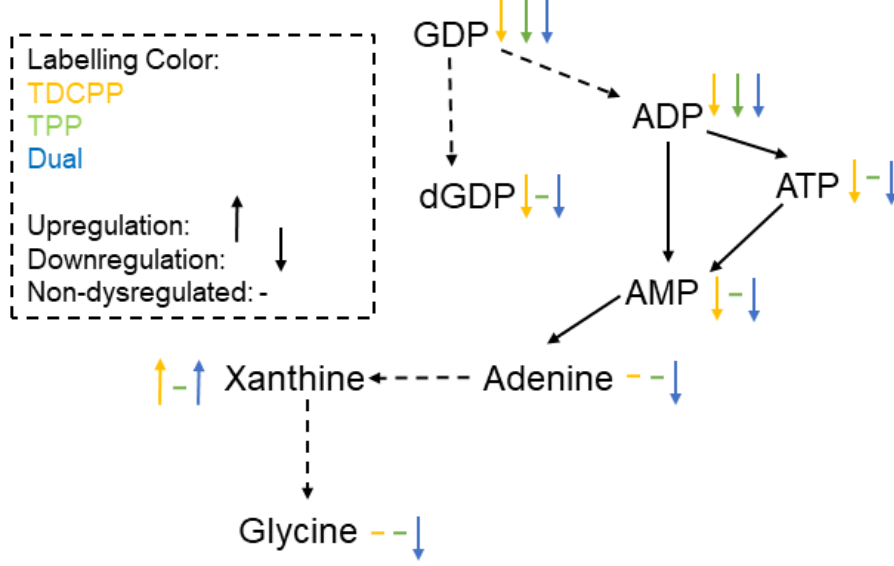

(a) Pyrimidine Metabolism

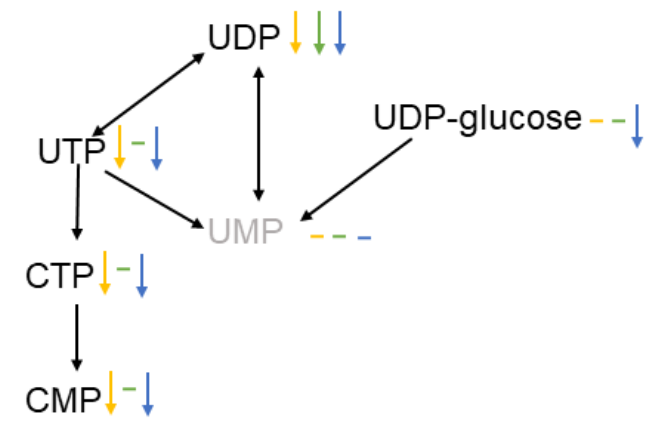

Figure S14. Dysregulated metabolites mapped to Purine Metabolism and Pyrimidine Metabolism. 
A. Alanine, aspartate and glutamate metabolism

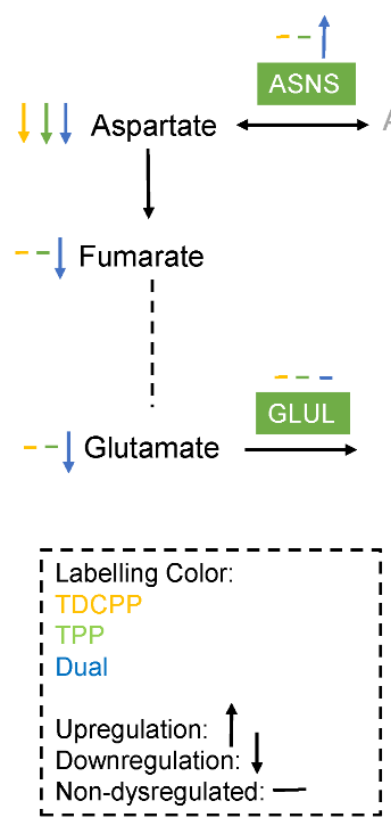

B. Aminoacyl-tRNA biosynthesis

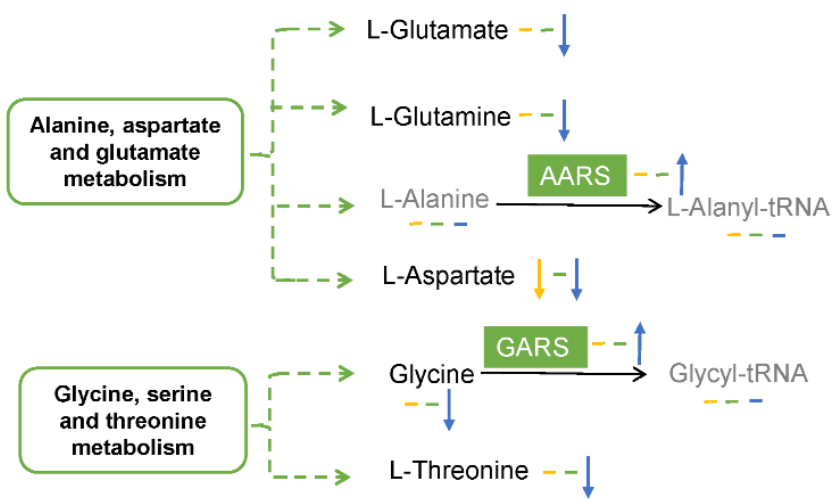

274 Figure S15. Dysregulated metabolites and genes expressions in A. "Alanine, aspartate and glutamate 275 metabolism" and B. "Aminoacyl-tRNA biosynthesis" in HepG2 under the exposure of TDCPP, TPP or 276 ASNS: Asparagine synthetase GLUL: Glutamine synthetase GFPT1: Glucosamine-fructose-6-phosphate aminotransferase isomerizing 1 AARS: Alanyl-TRNA Synthetase GARS: Glycyl-TRNA Synthetase CARS2: Cysteinyl-TRNA Synthetase 2

Dual. 


\section{Text S1. RNA extraction.}

283 Total RNA was extracted from the HepG2 using TRIzol® Reagent (Plant RNA Purification Reagent

284 for plant tissue) according the manufacturer's instructions (Invitrogen) and genomic DNA was removed 285 using DNase I (TaKara). Then RNA quality was determined by 2100 Bioanalyser (Agilent) and 286 quantified using the ND-2000 (NanoDrop Technologies). Only high-quality RNA sample $287(\mathrm{OD} 260 / 280=1.8 \sim 2.2, \mathrm{OD} 260 / 230 \geqslant 2.0, \mathrm{RIN} \geqslant 6.5,28 \mathrm{~S}: 18 \mathrm{~S} \geqslant 1.0,>2 \mu \mathrm{g})$ was used to construct sequencing library.

\section{Text S2. Library preparation, and Illumina Novaseq 6000 Sequencing.}

291 RNA purification, reverse transcription, library construction and sequencing were performed at 292 Shanghai Majorbio Bio-pharm Biotechnology Co., Ltd. (Shanghai, China) according to the 293 manufacturer's instructions (Illumina, San Diego, CA). RNA-seq transcriptome librariy was prepared 294 following TruSeqTM RNA sample preparation Kit from Illumina (San Diego, CA) using $1 \mu \mathrm{g}$ of total 295 RNA. Shortly, messenger RNA was isolated according to polyA selection method by oligo(dT) beads 296 and then fragmented by fragmentation buffer firstly. Secondly double-stranded cDNA was synthesized 297 using a SuperScript double-stranded cDNA synthesis kit (Invitrogen, CA) with random hexamer 298 primers (Illumina). Then the synthesized cDNA was subjected to end-repair, phosphorylation and 'A' 299 base addition according to Illumina's library construction protocol. Libraries were size selected for 300 cDNA target fragments of 200-300 bp on 2\% Low Range Ultra Agarose followed by PCR amplified using Phusion DNA polymerase (NEB) for 15 PCR cycles. After quantified by TBS380, paired-end RNA-seq sequencing library was sequenced with the Illumina Novaseq 6000(2×150bp read length).

\section{Text S3. Read mapping.}

The raw paired end reads were trimmed and quality controlled by SeqPrep (https://github.com/jstjohn/SeqPrep) and Sickle (https://github.com/najoshi/sickle) with default parameters. Then clean reads were separately aligned to reference genome with orientation mode using TopHat (http://tophat.cbcb.umd.edu/version2.1.1) ${ }^{1}$ software. The mapping criteria of bowtie was as follows: sequencing reads should be uniquely matched to the genome allowing up to 2 mismatches, without insertions or deletions. Then the region of gene were expanded following depths of sites and the operon was obtained. In addition, the whole genome was split into multiple $15 \mathrm{~kb}$ windows that share $5 \mathrm{~kb}$. New transcribed regions were defined as more than 2 consecutive windows without overlapped region of gene, where at least 2 reads mapped per window in the same orientation.

\section{Text S4. Differential expression analysis and Functional enrichment.}

316 To identify DEGs (differential expression genes) between two different samples, the expression level 
reads (FPKM) method. RSEM (http://deweylab.biostat.wisc.edu/rsem/) $)^{2}$ was used to quantify gene abundances. R statistical package software EdgeR (Empirical analysis of Digital Gene Expression in R, http://www.bioconductor.org/packages/2.12/bioc/html/edgeR.html) ${ }^{3}$ was utilized for differential expression analysis. In addition, GO functional enrichment and KEGG pathway analysis were DAVID Bioinformatic Resources 6.8 (https://david.ncifcrf.gov/) and KOBAS 3.0 (http://kobas.cbi.pku.edu.cn/) with a adjusted $p$-value $\leqslant 0.05$ and 0.01 .

Text S5. Solubility and metabolite measurement of compounds in culture medium and PBS buffer. To address the concern of compound solubility, we have collected the experimental solubility $\left(25{ }^{\circ} \mathrm{C}\right.$ $120{ }^{\circ} \mathrm{C}$ in water) information of all tested compounds from EPI Suite ${ }^{\mathrm{TM}}$ (https://www.epa.gov/tscascreening-tools/epi-suitetm-estimation-program-interface) and PubChem (https://pubchem.ncbi.nlm.nih.gov/). As presented in Table S3, the experimental solubility of some compounds is lower than dosing concentration in this study. Hence, we measured the actual concentrations of these chemicals (BADGE, TPP, TDCPP, B[a]p, Fluoranthene, Carbazole) in DMEM medium with $10 \%$ fetal bovine serum (FBS) to determine their actual concentrations and prepare another test using phosphate buffer ( $\mathrm{pH} 7.4$ ) alongside as comparison. After dosing the chemicals, the cell culture medium was incubated in cell incubator to reach the $\mathrm{CO}_{2}$ equilibrium at $37^{\circ} \mathrm{C}$. After 2 hours' incubation, the culture medium was rapidly centrifuged at $13.5 \mathrm{k} \mathrm{rpm}$ for $15 \mathrm{mins}$ to remove any particles and then the supernatant was transferred to a new Eppendorf tube. Subsequently, chemicals of BADGE, TPP and TDCPP were further spiked with methanol with a final volume ratio of $50 \%$ to precipitate the proteins. The supernatant was collected again after another centrifugation and spiked with internal standard (BCIPP-d12, TCEP-d12, BADGE-d6) for quantification using LC-QToF-MS. The quantification was conducted using internal standard based relative response factor method by integrating the abundance of the parent ions extracted from total ion chromatography. For the chemicals of $\mathrm{B}[\mathrm{a}] \mathrm{p}$, Fluoranthene and Carbazole, after incubation, centrifugation, transferring and spiking with internal standards Chrysene-d12 and Phenanthrene-d10, $1 \mathrm{~mL}$ ethyl acetate was added for liquid-liquid extraction (LLE). These three compounds were further analysed by GC-MS using single-ion monitoring method. ${ }^{4}$ After measuring the solubility in cell culture medium, all actual concentrations of aforementioned compounds have been determined and all the nominal concentration has been replaced as the measured values accordingly (Table S5).

348 Quantification of TPP, TDCPP and their metabolites (DPP, OH-TPP, BDCPP) along with PFOA, 349 BADGE, were performed on the LC-Q-ToF-MS. The electrospray ionization (ESI) was operated in positive mode except PFOA. Compounds were separated using Atlantis T3 column $((3 \mu \mathrm{m}, 2.1 \times 100$ $\mathrm{mm}$, Waters) with an injection volume of $5 \mu \mathrm{L}$. The LC mobile phases were water contained $5 \mathrm{mM}$ of ammonium acetate (A) and acetonitrile (B). The mobile phase flow rate was $0.3 \mathrm{~mL} / \mathrm{min}$ and the gradient was as follows: $1.5 \mathrm{~min}, 5 \% \mathrm{~B} ; 1.5-8 \mathrm{~min}, 95 \% \mathrm{~B}$ (linear); 8-10 min, 95\% B; 10-10.5 min, 5\% 
B (linear); 10.5-13.5 min, 5\% B. Carbazole, fluoranthene and $\mathrm{B}[\mathrm{a}] \mathrm{p}$ were quantified using a gas chromatograph (Agilent GC-6890 PLUS) and a mass selective detector (Agilent GC/MS 5973N) equipped with a $30 \mathrm{DB}-5 \mathrm{MS}$ used-silica column $(0.25 \mathrm{~mm}$ i.d. $\times 0.25 \mu \mathrm{m}$ film thickness; Agilent Technologies, Santa Clara, CA, U.S.A). The temperature program was: splitless injections at $280{ }^{\circ} \mathrm{C}$, the oven temperature was increased from $60{ }^{\circ} \mathrm{C}$ to $150{ }^{\circ} \mathrm{C}$ at a rate of $25^{\circ} \mathrm{C} / \mathrm{min}$ (hold $1 \mathrm{~min}$ ), then 15 ${ }^{\circ} \mathrm{C} / \mathrm{min}$ to $280^{\circ} \mathrm{C}$ (hold $1 \mathrm{~min}$ ), then $8^{\circ} \mathrm{C} / \mathrm{min}$ to $320^{\circ} \mathrm{C}$. In addition, one blank sample was included in each batch of samples analyzed. The other parameters could be found in Table S4 in SI.

Results in Table S3 show that TPP, TDCPP, B[a]p, Fluoranthene, Carbazole have saturated concentration. However, the solubility of most compounds in DMEM medium with $10 \%$ fetal bovine serum (FBS) is higher than phosphate buffer (pH 7.4). For instance, the solubility of TPP in DMEM medium with $10 \%$ FBS is approximately $70 \mu \mathrm{M}$ whereas its solubility in PBS is lower than $15 \mu \mathrm{M}$; the solubility of Fluoranthene in medium is more than 10 times higher than in PBS. Interestingly, the solubility of TDCPP is nearly identical between DMEM medium and PBS. In our earlier work, we also observed that some hydrophobic compounds did reach over-saturation in phosphate buffer ( $\mathrm{pH}$ 7.4), but the actual concentrations in DMEM medium were higher ${ }^{5}$. The enhanced solubility in DMEM medium could be a result of increased temperature $37^{\circ} \mathrm{C}$ in incubator and $\mathrm{pH}$ dependent deprotonation. Furthermore, the cell culture medium with $10 \%$ fetal bovine serum is rich in protein and lipids and might assist the dissolution of hydrophobic compounds. The capacity to bind to serum proteins can enhance the solubility of hydrophobic compounds and this phenomenon has been widely applied to poorly soluble drugs. ${ }^{6}$

\section{References}

1. Trapnell, C.; Pachter, L.; Salzberg, S. L., TopHat: discovering splice junctions with RNA-Seq. Bioinformatics 2009, 25, (9), 1105-1111. 
$3962 . \quad \mathrm{Li}, \mathrm{B}$.; Dewey, C. N., RSEM: accurate transcript quantification from RNA-Seq data with or 397 without a reference genome. BMC bioinformatics 2011, 12, (1), 323.

$398 \quad 3 . \quad$ Robinson, M. D.; McCarthy, D. J.; Smyth, G. K., edgeR: a Bioconductor package for 399 differential expression analysis of digital gene expression data. Bioinformatics 2010, 26, (1), 139-140.

$400 \quad 4 . \quad$ Fang, M.; Getzinger, G. J.; Cooper, E. M.; Clark, B. W.; Garner, L. V. T.; Di Giulio, R. T.;

401 Ferguson, P. L.; Stapleton, H. M., Effect-directed analysis of Elizabeth River porewater:

402 Developmental toxicity in zebrafish (Danio rerio). Environmental Toxicology and Chemistry 2014, 33, 403 (12), 2767-2774.

404 5. Peng, B.; Liu, M.; Han, Y.; Wanjaya, E. R.; Fang, M., Competitive Biotransformation Among 405 Phenolic Xenobiotic Mixtures: Underestimated Risks for Toxicity Assessment. Environmental Science 406 \& Technology 2019, 53, (20), 12081-12090.

$407 \quad 6 . \quad$ Buckley, S. T.; Fischer, S. M.; Fricker, G.; Brandl, M., In vitro models to evaluate the 408 permeability of poorly soluble drug entities: Challenges and perspectives. European Journal of 409 Pharmaceutical Sciences 2012, 45, (3), 235-250. 